\title{
A common pathomechanism in GMAP-210- and LBR-related diseases
}

\author{
Anika Wehrle, ${ }^{1}$ Tomasz M. Witkos, ${ }^{2}$ Judith C. Schneider, ${ }^{1}$ Anselm Hoppmann ${ }^{1,3}$ Sidney Behringer, ${ }^{1}$ \\ Anna Köttgen, ${ }^{3}$ Mariet Elting, ${ }^{4}$ Jürgen Spranger, ${ }^{1}$ Martin Lowe, ${ }^{2}$ and Ekkehart Lausch ${ }^{1}$ \\ 'Department of Pediatrics, Medical Centre-University of Freiburg, Faculty of Medicine, University of Freiburg, Freiburg, \\ Germany. ${ }^{2}$ Faculty of Biology, Medicine and Health, University of Manchester, Manchester, United Kingdom. ${ }^{3}$ Institute \\ of Genetic Epidemiology, Medical Centre-University of Freiburg, Faculty of Medicine, University of Freiburg, Freiburg, \\ Cermany. ${ }^{4}$ Department of Clinical Genetics, VU University Medical Center, Amsterdam, Netherlands.
}

Biallelic loss-of-function mutations in TRIP11, encoding the golgin CMAP-210, cause the lethal human chondrodysplasia achondrogenesis 1A (ACC1A). We now find that a homozygous splicesite mutation of the lamin $B$ receptor ( $L B R$ ) gene results in the same phenotype. Intrigued by the genetic heterogeneity, we compared CMAP-210- and LBR-deficient primary cells to unravel how particular mutations in LBR cause a phenocopy of ACC1A. We could exclude a regulatory interaction between LBR and GMAP-210 in patients' cells. However, we discovered a common disruption of Golgi apparatus architecture that was accompanied by decreased secretory trafficking in both cases. Deficiency of Colgi-dependent glycan processing indicated a similar downstream effect of the disease-causing mutations upon Golgi function. Unexpectedly, our results thus point to a common pathogenic mechanism in CMAP-210- and LBR-related diseases attributable to defective secretory trafficking at the Golgi apparatus.

Authorship note: AW, TMW, and JCS contributed equally to this work.

Conflict of interest: The authors have declared that no conflict of interest exists.

License: This work is licensed unde the Creative Commons Attribution 4.0 International License. To view a copy of this license, visit http:// creativecommons.org/licenses/ by/4.0/.

Submitted: March 19, 2018 Accepted: October 29, 2018 Published: December 6, 2018

\section{Reference information:} JCI Insight. 2018;3(23):e121150. https://doi.org/10.1172/jci. insight.121150.

\section{Introduction}

Recessive loss-of-function mutations in the lamin B receptor $(L B R)$ and the thyroid hormone receptor interactor 11 (TRIP11) genes cause lethal human chondrodysplasias with distinct phenotypes $(1,2)$. Owing to the dual functions of the LBR protein, which acts as both a receptor for lamin B and an enzyme of the cholesterol biosynthesis pathway, LBR-linked diseases are currently classified as laminopathies as well as disorders of cholesterol biosynthesis $(3,4)$. There is a remarkable genotype-phenotype correlation; depending on the state, nature, and site of mutation, the phenotypes range from asymptomatic to embryonic lethal $(3,5)$. In a heterozygous state, some, but not all, $L B R$ mutations cause Pelger-Huët anomaly (PHA, MIM 169400), a benign condition just marked by the hypolobulation of granulocyte nuclei (5). Compound heterozygous $L B R$ mutations were recently identified in PHA cases affected by mild skeletal anomalies and short stature $(6,7)$. Furthermore, an intermediate phenotype, displaying mental retardation and skeletal defects, was also described among the original PHA cases. There, a homozygous splice acceptor mutation leading to the skipping of exon 13 of $L B R$ was found (8). At the severe end, biallelic loss-of-function mutations of $L B R$ fatally disrupt skeletal development, resulting in the clearly recognizable clinical entity of hydrops-ectopic-calcification-moth-eaten (HEM), also known as Greenberg dysplasia (GRBGD, MIM 215140) (9). Interestingly, the very same heterozygous mutations found in PHA lead to GRBGD when both alleles are affected $(3,10)$. GRBGD is currently included in the chondrodysplasia punctata (CDP) group of diseases, which covers heterogeneous metabolic skeletal dysplasias with overlapping clinical features (2). Enzyme deficiencies in CDP are believed to converge on lipid biogenesis (11). Notably, similar to GRBGD, enzymes of cholesterol metabolism are also deficient in a number of CDP types. In keeping with a previous report of abnormal cholesterol metabolites in fetal GRBGD tissues (10), this finding argues in favor of GRBGD being in fact an inborn error of metabolism, the more so as recent data indicate that disease-associated LBR variants have reduced $3 \beta$-hydroxysterol $\Delta^{14}(\mathrm{C} 14$ sterol) reductase activity $(12$, 13). However, there is no genuine in vivo model faithfully recapitulating LBR-associated human disease (14-16). Hence, the relevance of these findings for disease development remains presently unclear, as all functional studies to date have been conducted in heterologous tumor cell lines $(4,14)$. The pathology of bone lesions in other CDP types is unknown as well, so this does not help in the determination of potential 
disease mechanisms in GRBGD (11). Therefore, despite some evidence for a physiological role of LBR in sterol biogenesis and inner nuclear membrane integrity, the pathophysiology of GRBGD and other LBR-related diseases remains largely obscure.

We report here on a case carrying what we believe is a novel homozygous splice site mutation of $L B R$. This mutation is predicted to cause a complete abrogation of both the lamina-binding function and the cholesterol reductase activity of LBR. On the basis of current knowledge, a recessive loss-of-function mutation of $L B R$ would be expected to cause GRBGD. On the contrary, hallmarks of GRBGD, such as a moth-eaten radiographic appearance of the long bones and ectopic calcification, were lacking in our case. Instead, the phenotype fully matched that of achondrogenesis 1A (ACG1A, MIM 200600), and thereby represents to our knowledge the first instance of this disease caused by mutations in $L B R$. ACG1A is considered to be one of the most severe human chondrodysplasias and has a specific clinical presentation (1). Since all reported cases of ACG1A to date are due to mutations in $\operatorname{TRIP11}(17,18)$, which encodes a golgin also known as GMAP-210 (Golgi-microtubule-associated protein, $210 \mathrm{kDa}$ ), we were curious to find out whether the striking clinical similarity of LBR-and TRIP11-associated achondrogenesis point to a common Golgi-related pathophysiology. Golgins are coiled-coil proteins that mediate vesicle tethering at the Golgi complex important for vesicular trafficking and Golgi organization $(19,20)$. However, there is conflicting evidence about the role of GMAP-210 in secretory protein transport (21-24). We therefore investigated the molecular consequences of disease-causing mutations in ACG1A-derived primary cells and found that not only GMAP-210, but also LBR, are indeed both required for efficient protein trafficking and processing in the secretory pathway. As secretory trafficking and cargo processing are of particular importance for extracellular matrix production by chondrocytes, our results can explain the developmental skeletal defects seen in ACG1A and in diseases caused by LBR deficiency.

\section{Results}

LBR-null mutations cause a phenocopy of ACG1A. We studied a male fetus from the second pregnancy of healthy consanguineous Turkish parents. The pregnancy was terminated at 21 weeks and 5 days because of intrauterine growth retardation and severe micromelia (Figure 1A). In addition, the fetus presented with short neck and trunk, narrow thorax, protuberant abdomen, hydrops, and distinctive radiographic features of ACG1A. Ossification of the skull, rib cage, and limbs was severely reduced. Further, radiographs demonstrated impaired ossification of the spine, the pedicles, and the sacrum. Scapulae were hypoplastic, the ossa ischium and the pubic bones were under-ossified, and an extreme shortening and broadening of the tubular bones was recognized (Figure 1B). Verified by independent experts (European Skeletal Dysplasia Network, http://www.esdn.org) (25), the clinical-radiographic features established a definitive diagnosis of ACG1A.

Sanger sequencing of TRIP11 failed to provide molecular confirmation for the clinical-radiographic diagnosis. With the assumption of a novel recessive skeletal disease, we used whole exome sequencing (WES) of the index case and his parents for unbiased disease gene identification. Thereby an undescribed homozygous $L B R$ c. $366+1 \mathrm{G}>\mathrm{T}$ variant at the boundary of exon 3 was detected. No additional segregating homozygous or heterozygous variants, known or predicted to impair gene function were found by comprehensive bioinformatics analysis. The nucleotide change in $L B R$ was validated by Sanger sequencing and cosegregation was confirmed by heterozygosity of the unaffected parents. Unaffected siblings were either carriers or wild type (Figure 1C). The variant was neither listed as a single nucleotide polymorphism (SNP) in public databases, nor found in an in-house exome database of matched ethnicity. In silico analysis predicted aberrant splicing with activation of an ectopic splice site within exon 3 (26).

To verify an effect on splicing, cDNA derived from primary fibroblasts of the fetus was analyzed by PCR with primers spanning the region of interest. We thereby identified a smaller amplicon of $542 \mathrm{bp}$ instead of $576 \mathrm{bp}$ expected for wild-type $L B R$ (Figure 1D). Sequencing of the PCR product implied the activation of an ectopic donor site, resulting in partial loss of exon 3 of $L B R$. In consequence, a frameshift leads to a premature translational stop codon in the open reading frame (ORF) of mutant $L B R$ (LBR p.E111S $f s^{*} 39$ ) that most likely induces nonsense-mediated mRNA decay (NMD) (27). In accordance, only a faint $L B R$-specific signal was seen using nonquantitative RT-PCR analysis. $L B R$ mRNA abundance was strongly reduced in primary fibroblasts obtained from the fetus (in the following referred to as ACG1A_1) (Figure 1D, Figure 2A, and Table 1). The early stop codon generated by the c.366+1G $>$ T mutation predicts a truncated, nonfunctional LBR protein. Accordingly, no LBR protein was detectable by immunoblotting 
A

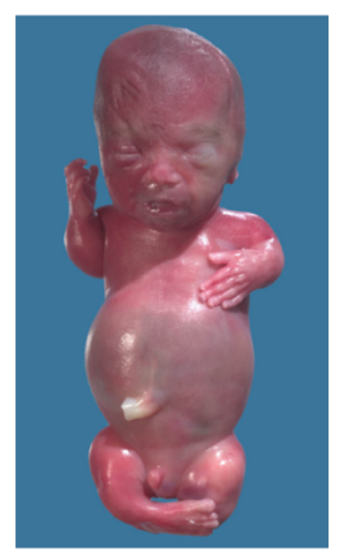

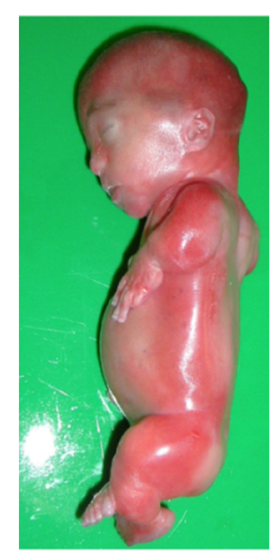
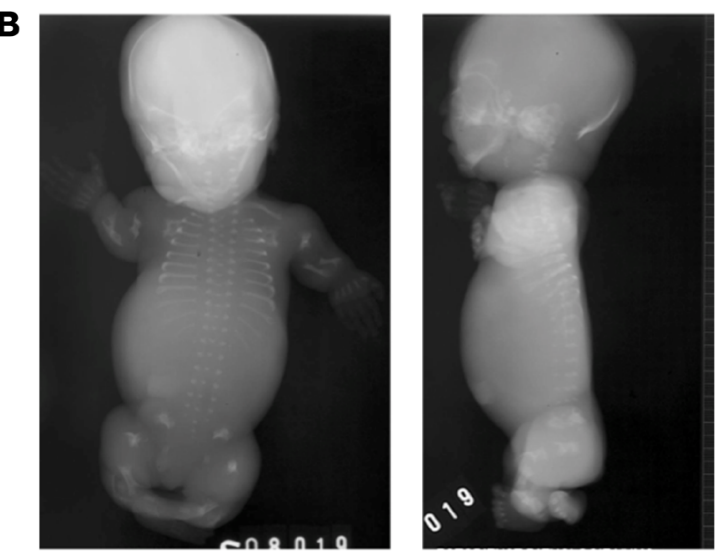

D

C

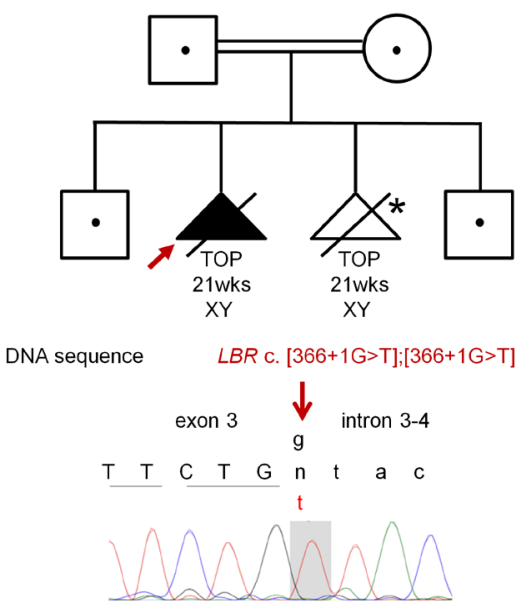

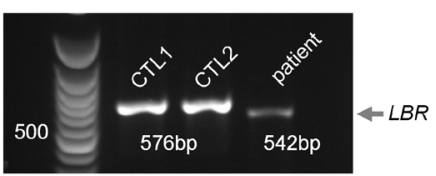

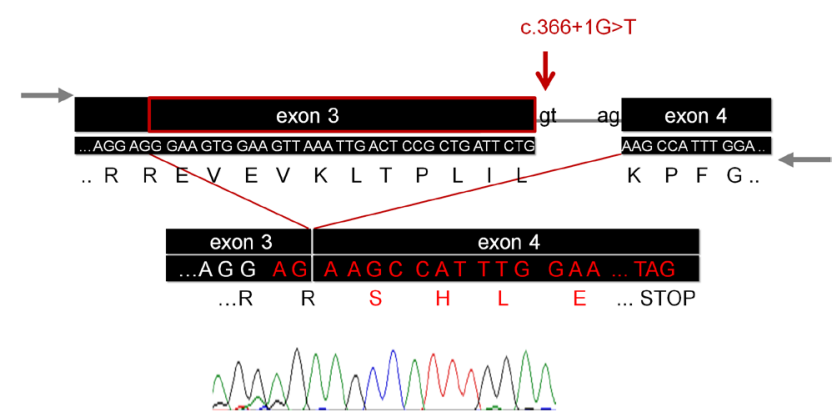

Figure 1. LBR mutations cause a phenocopy of achondrogenesis 1A (ACG1A). (A) Clinical photographs of the fetus at 21 weeks and 5 days, presenting with hydrops, shortened extremities, protruding abdomen, and short trunk. (B) Radiographs in frontal and lateral view show delayed ossification of spine and pelvis. Note the barrel-shaped thorax with horizontally oriented ribs and the extremely shortened long bones. (C) Sanger sequencing of genomic DNA demonstrates a homozygous mutation of the $L B R$ gene $(c .[366+1 g>t]$; $[366+1 g>t])$ that abrogates the splice donor site of exon 3 . The abridged pedigree above shows cosegregation of the LBR mutation; *termination of pregnancy (TOP) because of cardiac malformation. (D) Exon-spanning reverse transcription PCR analysis of $L B R$ (35 cycles) using cDNA derived from patient and matched control fibroblasts (CTL1, 2). Below: Schematic representation and electropherogram of the amplified/sequenced region and the splice mutation of $L B R$ (indicated by a red arrow) that causes a partial deletion of exon 3 , leading to a frameshift and premature termination codon in the open reading frame of $L B R$ (p.E112Sfs*39). Gray horizontal arrows indicate the relative position of primers (not to scale).

(Figure 2C), not even by overexposure of the membrane. This is in contrast to GRBGD, where residual aberrant LBR proteins comprising the lamina-binding domain are found (12). Thus, to the best of our knowledge our case represents the first true LBR-null phenotype.

There is no regulatory interaction between $L B R$ and GMAP-210. Since we had verified the $L B R$ mutation to be disease-causing in TRIP11-negative ACG1A, we were intrigued to unravel the pathophysiological basis of genetic heterogeneity. We first investigated whether a loss of $L B R$ had an effect on TRIP11 abundance. For this purpose, we compared primary LBR-deficient fetal fibroblasts (ACG1A_1) to fibroblasts of ACG1A cases with known TRIP11 nonsense mutations (ACG1A_2 and 3; Table 1) (17). Matched fibroblasts of healthy donors without mutations in TRIP11 and/or LBR served as controls (CTL 1 to 3). Interestingly, the TRIP11 mRNA concentration was approximately 2-fold higher in LBR-deficient cells (Figure 2B). However, since the amount of GMAP-210 protein was the same as in wild-type control (Figure 2D), we did not assume a significant effect ensuing from this upregulation. Vice versa, biallelic nonsense mutations of TRIP11 caused a strong reduction of TRIP11 mRNA and a complete loss of the GMAP-210 protein (Figure 2, B and D), while $L B R$ expression levels remained unaffected (Figure 2, A and C).

We next analyzed the intracellular distribution and abundance of LBR and GMAP-210 proteins by immunofluorescence microscopy. In keeping with previous reports $(12,13)$, control and GMAP-210-deficient cells showed a strong signal of the LBR confined to the nuclear envelope (Figure 3A). Less intense staining was 
A

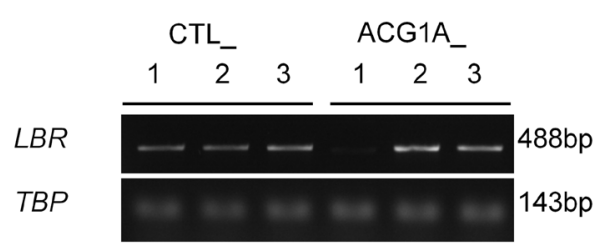

B

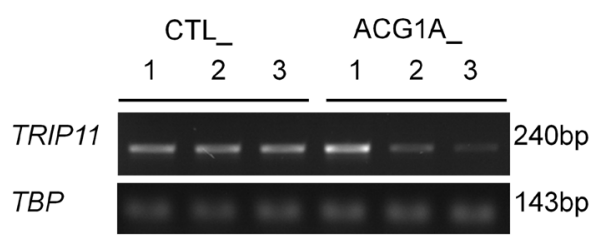

C

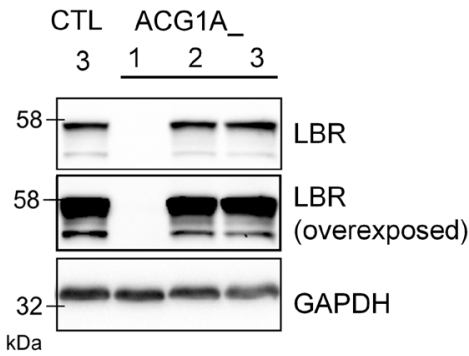

D

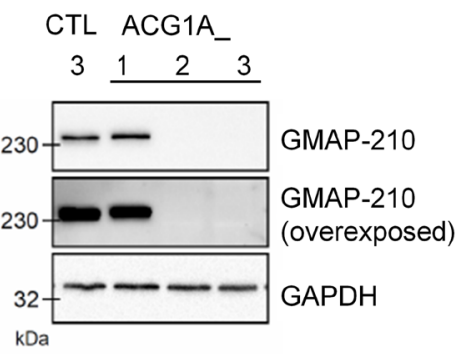

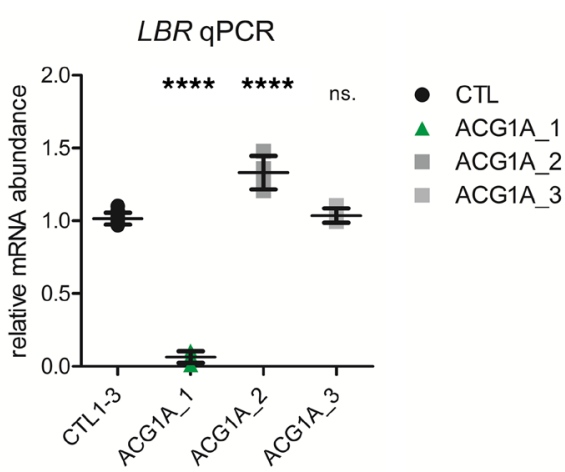

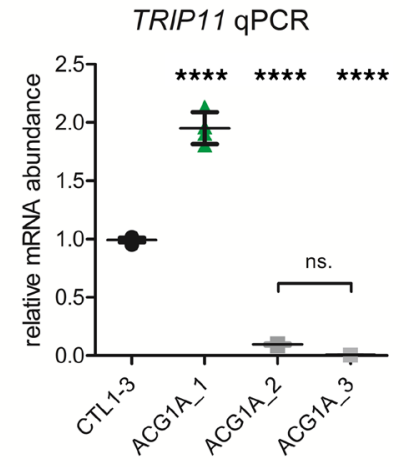

- CTL

- ACG1A_1

- ACG1A 2

- ACG1A_3

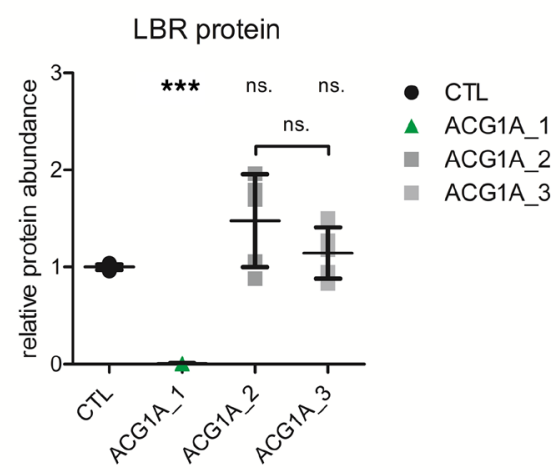

GMAP-210 protein

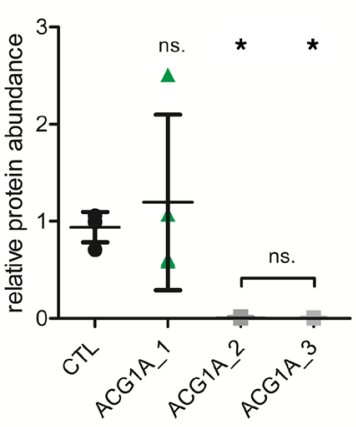

- cTL

- ACG1A 1

- ACG1A_2

- ACG1A_3
Figure 2. Analysis of LBR and TRIP11 mRNA and protein expression in achondrogenesis 1A (ACG1A) patient-derived primary cells. Semiquantitative reverse transcription PCR (left, 25 cycles), and quantitative real-time PCR (RT-qPCR) analysis (right) of (A) $L B R$, and (B) TRIP11 of cDNA derived from patient and control primary fibroblasts. ACG1A_1 carries the homozygous $L B R$ mutation described in this study, ACG1A_2 and 3 bear biallelic mutations of TRIP11; CTL_1 to 3 are matched wild-type controls. TBP was used for normalization; the same agarose gel is shown in $\mathbf{A}$ and $\mathbf{B}$, which are representative of 3 independent experiments $(N=3)$. For RT-qPCR results, the average value of the controls was set to $1(n=12)$. Horizontal lines represent the mean of quadruplicates $(n=4)$, error bars indicate SD. Statistical differences were assessed by 1-way ANOVA using Bonferroni's correction for multiple comparisons; ns., not significant. **** $P<0.0001$. Immunoblots of (C) LBR and (D) CMAP-210 protein in whole-cell lysates of patients and controls; GAPDH staining indicates total protein loading and was used for normalization in quantitative signal analysis (right). Horizontal lines represent the mean of triplicate quantitative blot signal analyses $(n=3)$, error bars indicate SD. Statistical differences were assessed by 1-way ANOVA using Bonferroni's correction for multiple comparisons; ns., not significant. ${ }^{*} P \leq$ $0.05,{ }^{* *} P<0.001$. See complete unedited blots in the supplemental material.

detected in the nuclear matrix, in the nucleoli, and throughout the endoplasmic reticulum (ER). In contrast, LBR staining was absent from the fibroblasts of ACG1A_1, confirming the loss of the LBR protein and the specificity of the antibody (Figure 3A). Lack of LBR did not change the intracellular localization of GMAP210, as the intense cis-Golgi-specific staining in ACG1A_1 cells was not different from controls (Figure 3B). As expected, no GMAP-210 signal was detected in the 2 ACG1A cases bearing biallelic nonsense mutations of TRIP11 (Figure 3B). Importantly, cis-Golgi staining by anti-GM130 antibodies revealed that the absence of GMAP-210 results in severe compaction of the Golgi apparatus, as reported previously (28) for transient 
Table 1. Clinical and genetic data of the analyzed achondrogenesis $1 \mathrm{~A}$ cases

\begin{tabular}{ccccccccc}
\hline Patient & Diagnosis & Origin & Consanguinity & Mutations & Status & Location & Predicted amino acid changes & Reference \\
1 & ACG1A & Turkish & Yes & $\begin{array}{c}\text { LBR } \\
\text { homozygous }\end{array}$ & $\begin{array}{c}\text { Int3-4 } \\
\text { p.[(Glu112Serfs*39)];[(Glu112Serfs*39)] }\end{array}$ This \\
study
\end{tabular}

ACG1A, achondrogenesis, type 1A; Int, intron; Ex, exon.

knockdown experiments in generic cell lines (Figure 3B). To the best of our knowledge, this study is the first to investigate the consequences of disease-specific TRIP11 mutations in patient-derived primary cells. Our observations support the view that GMAP-210 is an important structural component of the Golgi complex in humans (23). Taken together, our results show that loss-of-function mutations of $L B R$ had no apparent effect on GMAP-210, thereby excluding the possibility that the associated ACG1A phenotype is due to a downregulation or mislocalization of GMAP-210.

LBR-deficient cells are sensitive to cholesterol restriction. Because GRGBD-associated mutations inactivate the $\mathrm{C} 14$ sterol reductase domain of LBR and lead to an intracellular accumulation of precursors in the cholesterol biosynthesis pathway $(10,13,29)$, we analyzed the sterol composition of ACG1A_1 cells grown in lipoprotein-depleted medium. As described previously for GRGBD (10), gas chromatography/mass spec-

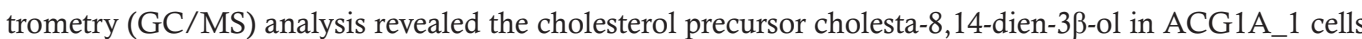
that was not detectable in matched healthy controls (Figure 4A). We thus concluded that both GRBGDand ACG1A-associated $L B R$ mutations cause a loss of sterol reductase function.

As C14 sterol reductase deficiency is known to impair de novo sterol biosynthesis in humans $(12,13)$, we further investigated whether ACG1A_1 cells are auxotrophic and depend on the delivery of exogenous cholesterol for growth and viability. Cellular cholesterol levels can be specifically manipulated by the addition of cyclodextrin complexes to conditioned media (30). The high affinity of cyclodextrins for cholesterol can be not only used to remove cholesterol from biological membranes, but also to generate cholesterol inclusion complexes that donate cholesterol and thereby increase cellular cholesterol levels (31). A complete knockout of $L B R$ has been shown to decrease viability of HeLa cells under cholesterol-restrictive growth conditions (12). In order to determine if C14 sterol reductase-deficient primary cells are auxotrophic as well, ACG1A_1 and control cells were grown for 48 hours in serum-free DMEM supplemented either with methyl- $\beta$-cyclodextrin $(\mathrm{M} \beta \mathrm{CD})$ to deplete cholesterol, or with $\mathrm{M} \beta \mathrm{CD}$ :cholesterol complexes to replicate cholesterol-proficient conditions in vivo. Compared with control cells, we observed a higher number of ACG1A_1 fibroblasts exhibiting cell rounding and detachment under cholesterol depletion, which indicates a higher susceptibility to cholesterol restriction (Figure 4B). Accordingly, addition of MBCD:cholesterol-saturated complexes to the medium rescued the observed phenotype in ACG1A_1 cells (Figure 4B). LBR was also rate limiting for proliferation under cholesterol-restrictive conditions. While controls showed exponential growth in lipid-depleted medium, the growth rate of ACG1A_1 cells progressively declined to zero from days 5 to 9 , consistent with growth arrest (Figure 4, C and D). Importantly, proliferation and viability were restored by the addition of cholesterol to the medium of ACG1A_1 cells. Hence, ACG1A-associated LBR mutations cause an error of metabolism characterized by intracellular accumulation of unreduced sterol derivatives and impaired de novo synthesis of cholesterol, which reduces cell proliferation and viability.

Nuclear envelope integrity is preserved in LBR-mutant human primary cells. To analyze intracellular compartments that may be affected in ACG1A, we performed high-resolution imaging studies, focusing on the nucleus, the ER, and the Golgi apparatus as the key intracellular locations of the LBR and GMAP-210 proteins. LBR has long been implicated in nuclear envelope integrity and anchoring to the nuclear lamina, as documented by $L B R$ mutations leading to nuclear hyposegmentation of granulocytes (Pelger-Huët anomaly) (32-35). The nuclear envelope comprises outer and inner membranes that enclose the luminal space. The inner nuclear membrane is lined with lamins and lamin-binding membrane proteins, such as LBR, which constitute the lamina $(3,34,36)$. The lamina connects to the nucleoplasm and controls critical nuclear functions, including mitosis, meiosis, and apoptosis, but also chromatin conformation and thereby 
A

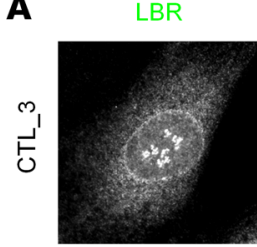

lamin_B
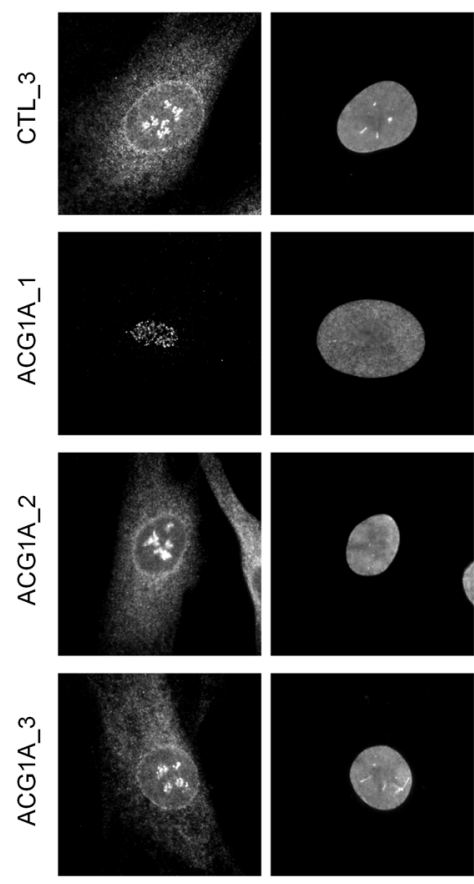

B
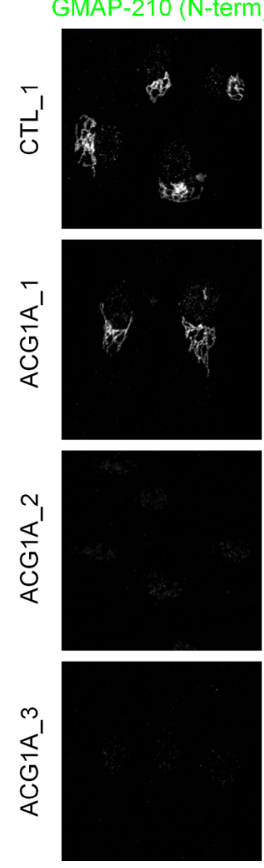
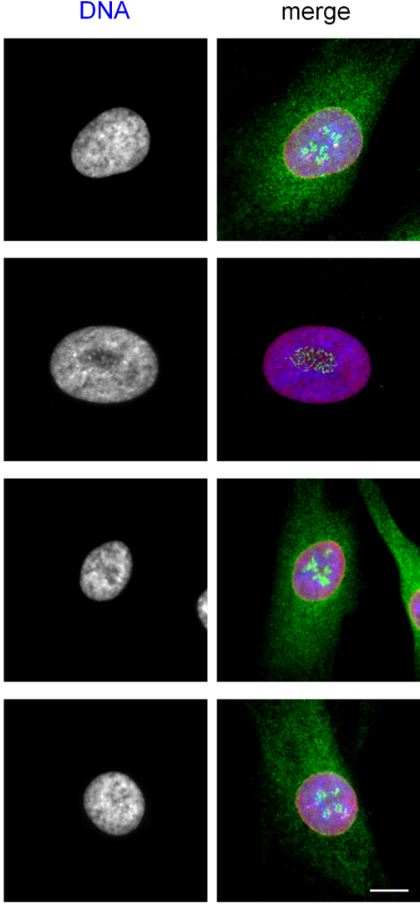

Figure 3. Loss of LBR or GMAP-210 in achondrogenesis 1A (ACG1A) patient-derived primary cells. Immunofluorescence microscopy of patient and control fibroblasts; ACC1A_1 carries the homozygous $L B R$ mutation described in this study, ACG1A_2 and 3 bear biallelic mutations of TRIP11; CTL_1 and 3 are representative matched wildtype controls. Cells were grown on coverslips, fixed, and costained with (A) LBR- and lamin B-specific antibodies, or (B) using antibodies directed against GMAP-210 and the cis-Golgi marker GM130. Nuclear DNA was stained with Hoechst 33342 dye. White lines indicate where red, green, and blue (RCB) fluorescence was quantified; RGB profile plots of the sections are shown on the right. Scale bars: $10 \mu \mathrm{m}$.

transcriptional activity (4). A laminar defect would therefore likely have severe consequences for basic cellular functions and is believed to be pathogenic in GRBGD (4).

However, alterations of the nuclear shape and envelope were neither observed in granulocytes of a fetal blood smear collected upon autopsy, nor in ACG1A_1 fetal fibroblasts by transmission electron microscopy (TEM) (Supplemental Figure 1A; supplemental material available online with this article; https:// doi.org/10.1172/jci.insight.121150DS1). As expected, a regular nuclear morphology was detected in TRIP11-mutant cells as well (Supplemental Figure 1A). In all analyzed cells, both nuclear membranes appeared correctly folded, and the ultrastructure of the luminal space and the intermembrane distance between the inner and the outer membrane were normal (Supplemental Figure 1B). This is in line with 
A
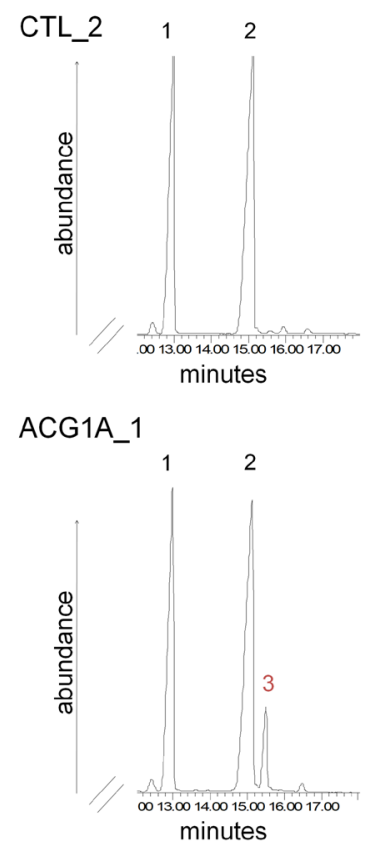

C

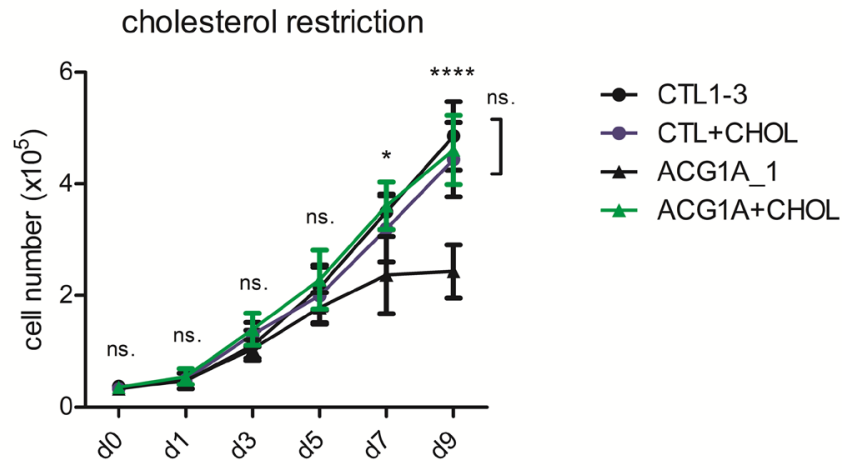

B

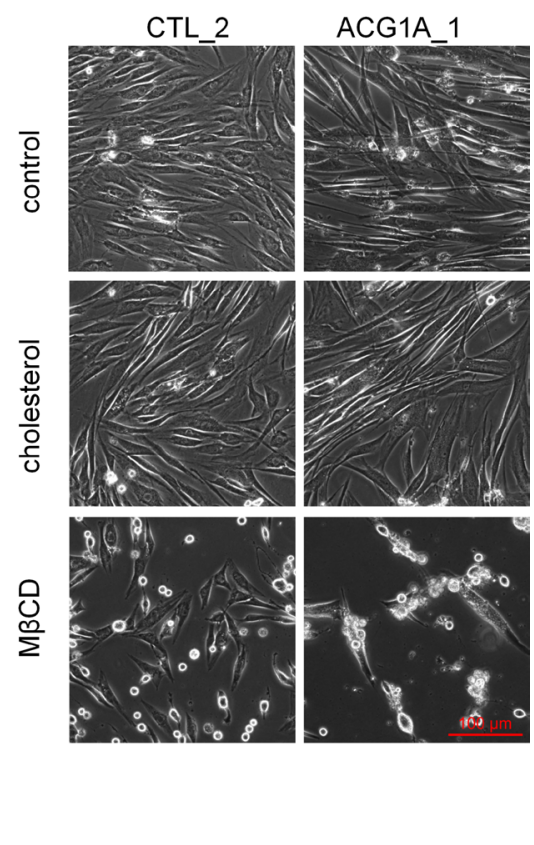


A
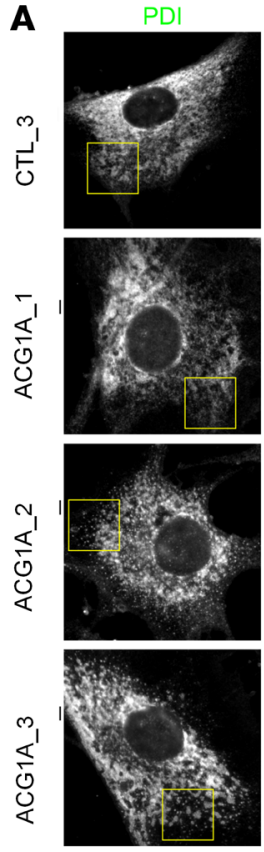

B
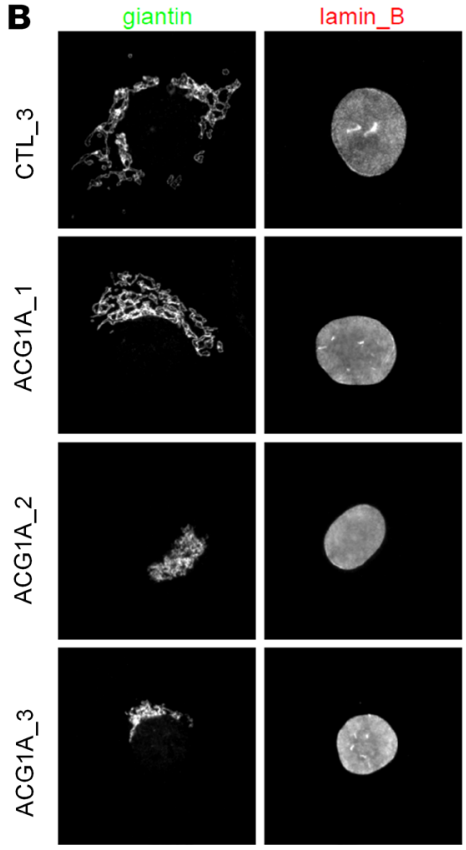

PDI (zoom)
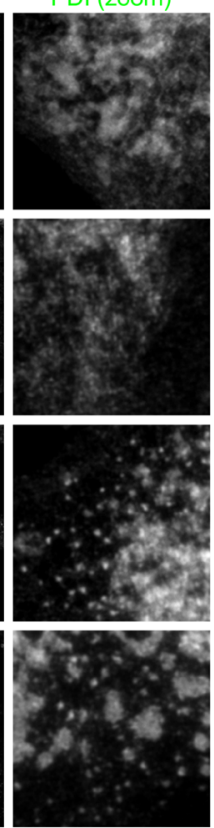

Iamin B
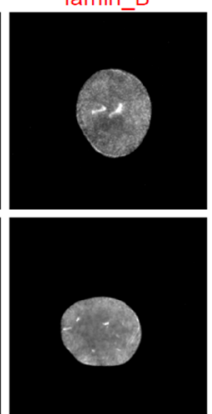

p230
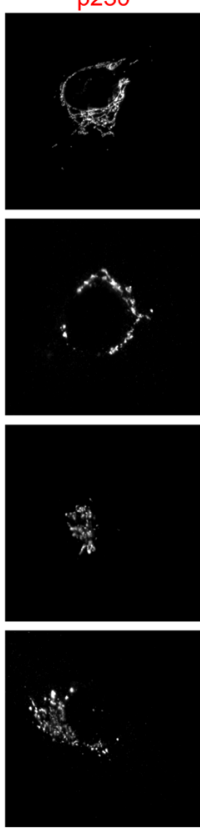

DNA
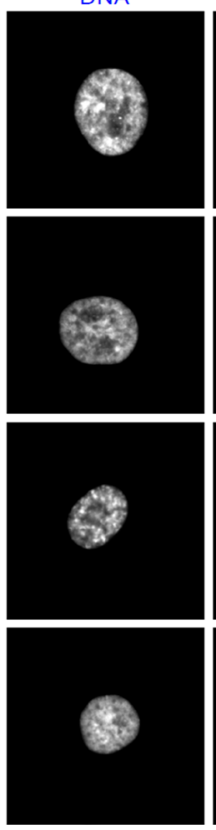

DNA/merge
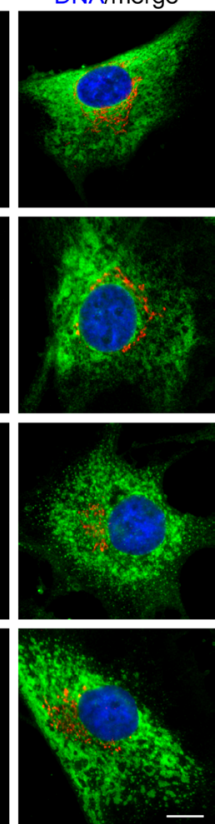

merge
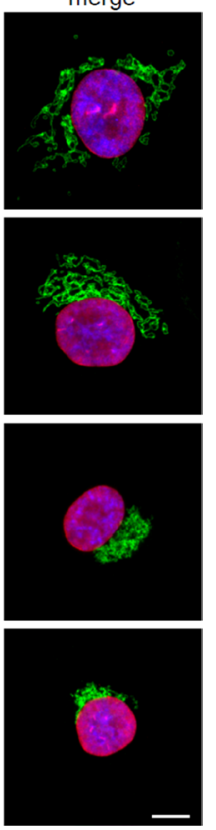

Figure 5. Endoplasmic reticulum (ER) and Golgi morphology in achondrogenesis 1A (ACG1A). Immunofluorescence microscopy of patient and control fibroblasts; ACG1A_1 carries the homozygous $\angle B R$ mutation described in this study, ACG1A_2 and 3 bear biallelic mutations of TRIP11; CTL_3 is representative of matched wild-type controls $(n=3)$. Cells were grown on coverslips, fixed, and (A) costained with antibodies directed against the ER marker protein disulfide isomerase (PDI) and the trans-Golgi protein p230, or (B) using antibodies directed against the cis- and medial-Golgi protein giantin and the LBR ligand lamin B. Nuclear DNA was stained with Hoechst 33342 dye. Scale bars: $10 \mu \mathrm{m}$.

membrane from the outer nuclear envelope, mutant variants of LBR induce an expansion of the ER in some cell types $(36,37)$. GMAP-210 is localized at the ER-Golgi intermediate compartment (ERGIC) and cis-Golgi and mediates trafficking processes in the anterograde (ER to Golgi) and retrograde (Golgi to ER) direction $(23,28,38)$. As previous ultrastructural analysis of Trip11-mutant mice has demonstrated swollen ER cisternae (17), we next investigated whether ACG1A-asssociated mutations may cause a common ER pathology. Protein disulfide isomerase (PDI) is an ER-resident chaperone with disulfide exchange activity (39). We used PDI staining to assess ER morphology in ACG1A. A normal ER structure comprising a mix of ER sheets and tubular structures with the ER-encircling lamina was observed in control and ACG1A_1 cells by immunofluorescence microscopy (Figure 5A). However, higher-resolution imaging using TEM demonstrated a swollen and less reticular ER in cells lacking LBR (Supplemental Figure 2). An irregular ER morphology was also detected by immunofluorescence microscopy in cells lacking GMAP-210, where there appeared to be less continuous ER in the cell periphery, with reduced number of ER sheets and 
tubules and an increased number of ER foci (Figure 5A, insets). Although these changes may correspond to the ultrastructural changes reported for Trip11-mutant mice (17), TEM was not sufficient to confirm comparable structural alterations in fibroblasts. The ER phenotype of TRIP11-deficient human cells therefore merits further in-depth investigation.

We also took a closer look at compartments involved in traffic between the ER and the Golgi. Coat protein complex II (COPII) operates in ER exit of cargo and is localized to specialized regions of the ER known as ER exit sites (ERES). In mammalian cells, cargo then passes through the ERGIC en route to the Golgi $(40,41)$. No substantial differences between patient-derived cells and controls were detected when we analyzed the intracellular distribution of ERGIC53 and SEC13, specific markers of ERGIC and ERES (Supplemental Figure 3). Importantly, in spite of the observed alterations in ER morphology, the abundance and organization of ERES and ERGIC structures was not markedly affected in LBR- or GMAP-210-deficient cells. Taken together, our findings make a common ER-related pathogenic mechanism in ACG1A unlikely. We therefore focused on a possible Golgi phenotype of LBR-mutant cells.

ACG1A-associated mutations cause common structural alterations of the Golgi apparatus. RNAi-mediated depletion of GMAP-210 in generic cell models has been reported to cause Golgi ribbon compaction and loss of Golgi ultrastructure (23), supporting the hypothesis that a Golgi-related pathogenic mechanism underlies ACG1A. This view is further strengthened by findings in Trip11-knockout mice, where a disrupted Golgi is observed in certain cell types $(17,42)$. As expected, mutations of TRIP11 in patient-derived primary cells caused a disruption of Golgi architecture, with compaction and breaking of the Golgi ribbon (Figure 3B, and Figure 5, A and B). TEM revealed a dramatic loss of cisternal stack architecture and the accumulation of numerous vesicular profiles in the Golgi region (Figure 6, A and B). Although fluorescence microscopy demonstrated no obvious change in Golgi organization in ACG1A_1 cells, where the Golgi ribbon retained its typical appearance (Figure 3B, and Figure 5, A and B), the higher resolution of TEM disclosed a disruption of Golgi ultrastructure similar to that seen upon loss of GMAP-210 (Figure 6, A and B). LBR deficiency led to a marked loss of stacked cisternae and the accumulation of vesicular profiles in the Golgi region. This vesiculation was not seen in matched controls, which exhibited an intact Golgi ultrastructure with clear, stacked cisternae and associated transport vesicles (Figure 6, A and B). Aberrant Golgi vesiculation in ACG1A_1 cells was an unexpected observation, since LBR is strictly confined to other intracellular compartments (Figure 3A) and has no known functional connection to the Golgi. However, it has previously been shown that cellular cholesterol levels are critical for Golgi morphology, and both overloading and depletion may induce vesiculation and dispersal of vesicles $(43,44)$. Importantly, exogenous cholesterol rescued the structural alterations of the Golgi apparatus in ACG1A_1 cells (Supplemental Figure 4, A and B). Given the enzymatic activity of LBR as a C14 sterol reductase, we therefore assumed that the intracellular accumulation of unreduced cholesterol metabolites [i.e., cholesta-8(9),14-

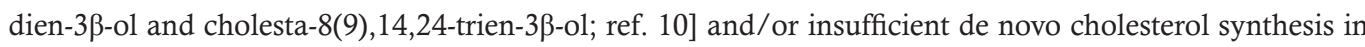
LBR-deficient cells are responsible for the Golgi phenotype $(12,13)$.

Secretory traffic and glycan processing by the Golgi complex are impaired in LBR-and GMAP-210-deficient cells. We assumed that the severe structural Golgi alterations in ACG1A reflect perturbed secretory trafficking at this organelle. Indeed, RNAi-mediated depletion of GMAP-210 has previously been shown to reduce secretory traffic in human cells (23). To measure bulk secretory protein trafficking in ACG1A patient-derived primary cells, we used a pulse-chase approach with radiolabeled amino acids $(23,45)$. Secretion of newly synthesized proteins into the extracellular space was significantly reduced in GMAP-210-deficient cells, indicating a delay in secretory protein transport to the plasma membrane (Figure 7, A and B, and Supplemental Figure 5). Reduced secretion was also observed in ACG1A_1 fibroblasts, albeit to a lesser extent than in GMAP-210-deficient cells (Figure 7, A and B). Interestingly, the profiles of secreted proteins differed between controls and ACG1A, but also slightly between GMAP-210 - and LBR-deficient cells (Figure 7A). Thus, the trafficking defect may affect cargo proteins differently, with considerable overlap in ACG1A.

Golgi trafficking ensures correct posttranslational modification of proteins, and disruption of this process often manifests as altered cargo protein glycosylation. Loss of Gmap-210 in mouse fibroblasts has previously been shown to impair glycosylation of specific cargoes (17). We therefore analyzed glycan processing in ACG1A patient cells by means of lysosome-associated membrane proteins (LAMPs), which are sensitive indicators of Golgi glycosylation capacity. LAMPs are lysosomal transmembrane proteins that undergo extensive glycosylation by the traditional N- and O-linked ER-to-Golgi glycan biosynthesis pathways (46). 
A
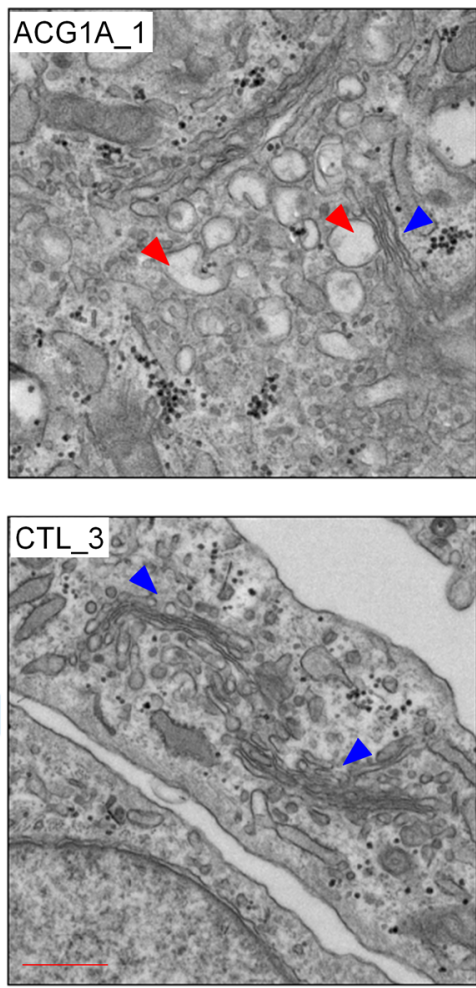
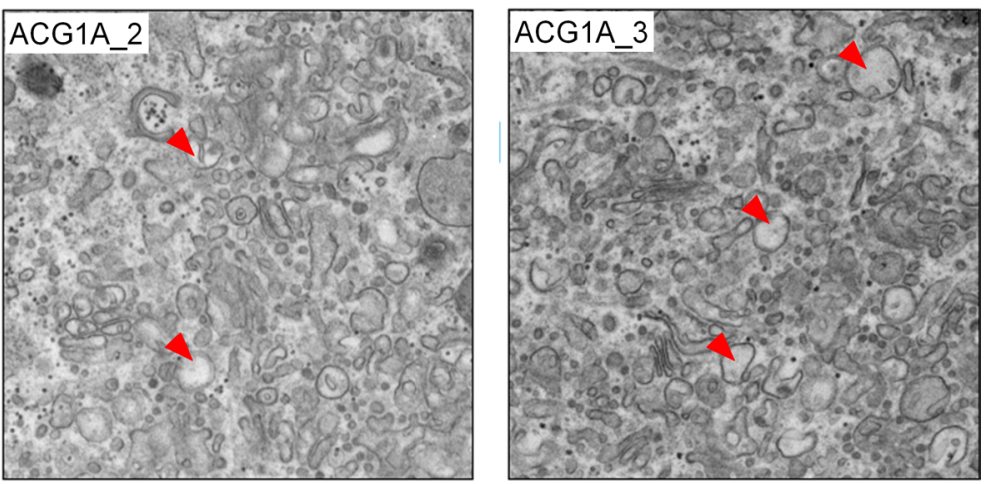

B

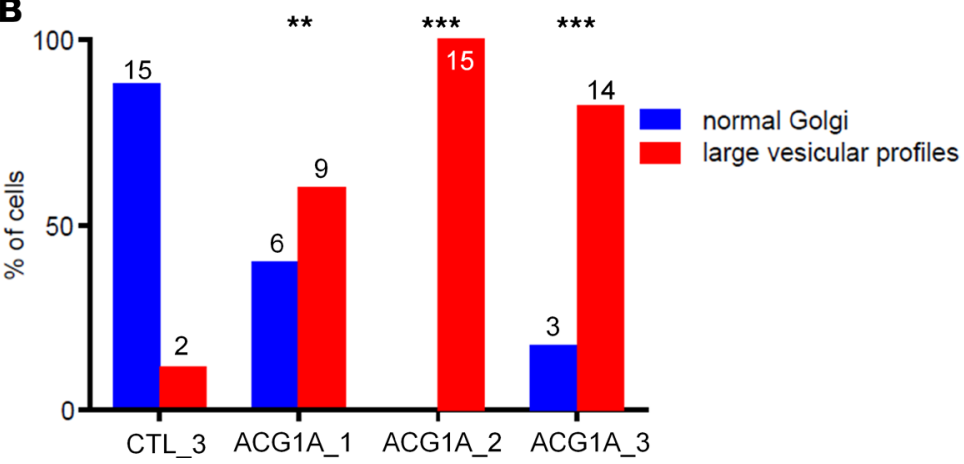

Figure 6. Abnormal vesiculation at the Golgi apparatus is a common cellular phenotype in achondrogenesis 1A (ACG1A). (A) Transmission electron microscopy of patient and control fibroblasts; ACG1A_1 carries the homozygous LBR mutation described in this study, ACG1A_2 and 3 bear biallelic mutations of TRIP11; CTL_3 is representative of 3 matched wild-type controls $(n=3)$. Red arrowheads mark large vesicular profiles, blue arrowheads normal Golgi stacks in ACG1A_1 and control cells. Scale bar: $500 \mathrm{~nm}$. (B) Quantification of cells with abnormal vesiculation, defined as peri-Golgi circular profiles with a diameter of $>100 \mathrm{~nm}$ (red bars) compared with cells with normal Golgi morphology (blue bars); the $y$ axis shows the percentage of respective phenotypes, and the individual cell counts are indicated above the bars. The significance of differences in the number of cells with large vesicular profiles was compared using a $\chi^{2}$ test; ${ }^{* *} P<0.01 ;{ }^{* *} P<0.001$.

In addition, several of the $\mathrm{N}$-linked adducts are modified by long poly-N-acetyl-lactosamines added exclusively in the Golgi apparatus (46). The molecular mass of the polypeptide backbone of LAMP1 and LAMP2 is approximately $40-45 \mathrm{kDa}$ and increases 3-fold to approximately $120 \mathrm{kDa}$ after glycosylation (47). Glycosylation of LAMP1 and LAMP2 was assessed by Western blot, and demonstrated aberrant electrophoretic mobility patterns in all ACG1A cells (Figure 7, C and D). As compared with wild-type cells, a lower average molecular weight range indicated hypoglycosylated species of both LAMP proteins in mutant primary fibroblasts, consistent with an incomplete modification of proteins in the Golgi. Further, we recognized additional low-molecular-weight, immature LAMP species in LBR- and GMAP-210-deficient cells, which are normally not detectable because they consist of short-lived intermediate products synthesized in the ER (Figure 7, C and D) (48). Notably, we also detected an increase in hypoglycosylated immature LAMPs when cholesterol was depleted from wild-type control cells by M $\beta C D$ (Supplemental Figure 6A) (30). Vice versa, supply of exogenous cholesterol to lipoprotein-starved ACG1A_1 cultures restored the glycosylation of immature LAMP species (Supplemental Figure 6B).

To corroborate that a functional Golgi defect in LBR-deficient cells solely results from the loss of sterol reductase activity of the protein, we next transiently overexpressed different LBR constructs in ACG1A_1 cells. The GRBGD-associated LBR mutations p.N547D and p.R583Q substitute amino acids highly conserved in homologous $\mathrm{C} 14$ sterol reductases and have recently been shown to selectively abrogate the binding capacity of the enzymatic core for reduced nicotinamide adenine dinucleotide phosphate (NADPH), a cofactor absolutely required for the reduction of sterol substrates by LBR (12). While the GRBGD-associated LBR missense mutants are hence enzymatically dead, the other functions and properties of the protein remain unaffected, in particular the lamin/chromatin interaction $(12,13)$. Accordingly, all exogenous LBR proteins transiently overexpressed in ACG1A_1 cells showed similar abundance and the same subcellular distribution as the endogenous protein in 
A

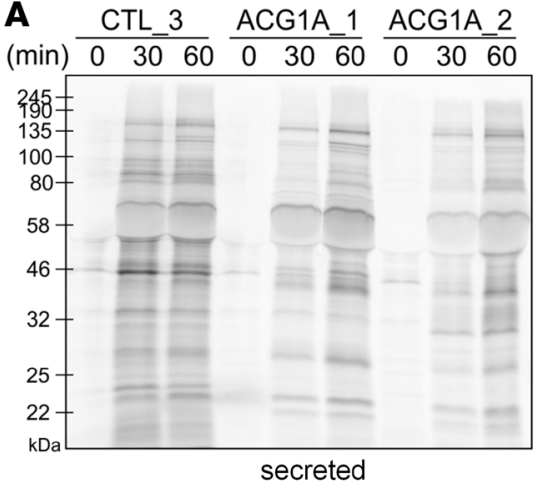

C $\mathrm{CTL}_{-}$ACG1A

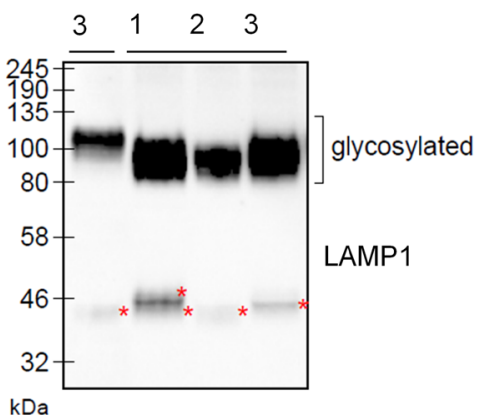

B

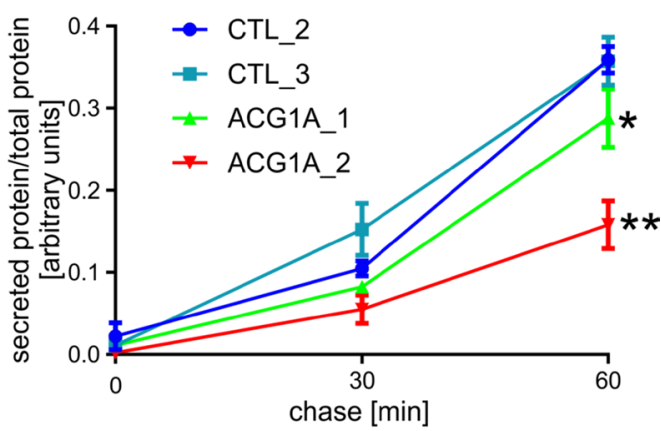

D

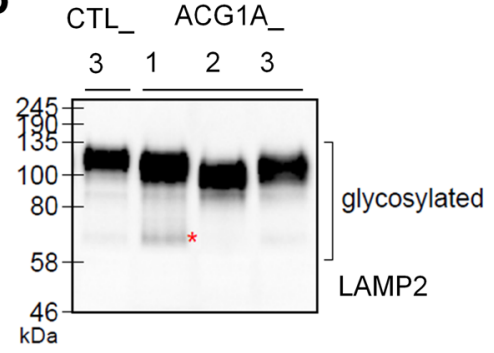

Figure 7. Reduced secretory trafficking and defective glycoprocessing in achondrogenesis 1A (ACG1A). Patient and control fibroblasts were starved for 1 hour in methionine- and cysteine-free medium and pulsed for 20 minutes with fresh starvation medium containing $50 \mu \mathrm{Ci} / \mathrm{ml}\left[{ }^{35} \mathrm{~S}\right]$ methionine and $\left.{ }^{35} \mathrm{~S}\right]$ cysteine protein-labeling mix. Cells were chased in medium containing unlabeled methionine and cysteine for 0,30 , or 60 minutes. Proteins secreted to the medium were precipitated and lysed. (A) Radiolabeled secreted proteins in trichloroacetic acid precipitates were subjected to denaturing gel electrophoresis and visualized by phosphorimaging. ACG1A_1 carries the homozygous $L B R$ mutation described in this study, ACG1A_2 bears biallelic mutations of TRIP11; CTL_2 and 3 are matched wild-type controls $(n=2)$. (B) Ratio of secreted-to-total proteins at the indicated time points. Data represent mean \pm SEM of 3 independent experiments $(n=3$; one-way ANOVA with Dunnett's multiple comparisons test). ${ }^{*} P \leq 0.05$, ${ }^{*} P<0.01$. (C) Immunoblot analyses of LAMP1, and (D) LAMP2 proteins in wholecell lysates of ACG1A patient and control fibroblasts. Membranes were overexposed to detect weaker signals. Low-molecular-weight intermediate glycosylation products of LAMP1 in ACG1A_1 are marked by a red asterisk. Similar, but faster migrating protein species are visible in ACG1A_3 (red asterisk). Non-glycosylated forms are present at very low steady state levels in CTL_3 and ACG1A_2 cells. As for LAMP1, the mature LAMP2 proteins in ACC1A run at a lower molecular weight range than in controls. An intermediate product is present in ACG1A_1 cells ( 60 kDa, red asterisk).

wild-type fibroblasts (Figure 3A), with a strong signal confined to the nuclear envelope and less intense staining of the nuclear matrix, the nucleoli, and throughout the ER (Figure 8A). Despite their correct intracellular localization, enzymatically inactive LBR mutants failed to restore normal glycan processing. In contrast, overexpression of wild-type LBR resulted in a strong reduction of immature LAMP2 species. Importantly, only the restitution of enzymatically competent LBR shifted highmolecular-weight LAMP2 species in ACG1A_1 towards the larger forms seen in wild-type fibroblasts, indicating normal protein maturation by Golgi-resident glycosyltranferases (Figure 8B). Thus, rescue of the functional Golgi defect in LBR-deficient cells is exclusively mediated by the C14 sterol reductase function of LBR.

In summary, we conclude that recessive loss-of-function mutations in LBR and in TRIP11 cause a common structural and functional defect of the Golgi complex (Figure 9). As chondrogenesis and ossification critically depend on the synthesis of numerous highly glycosylated proteins, such as the extracellular matrix (ECM) proteoglycans, our findings likely represent the shared pathogenic basis of skeletal diseases caused by LBR or GMAP-210 deficiency. 
A
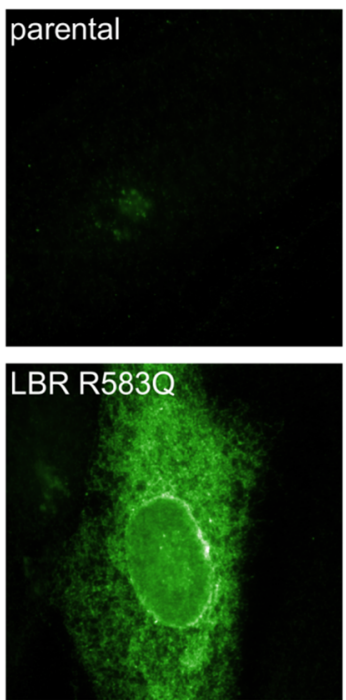

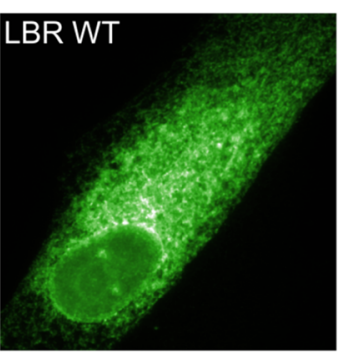

LBR N547D

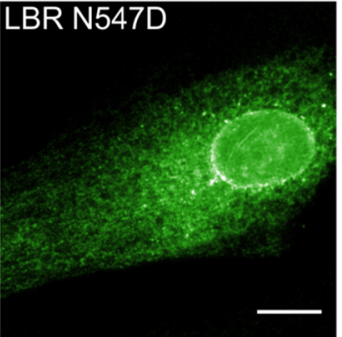

B

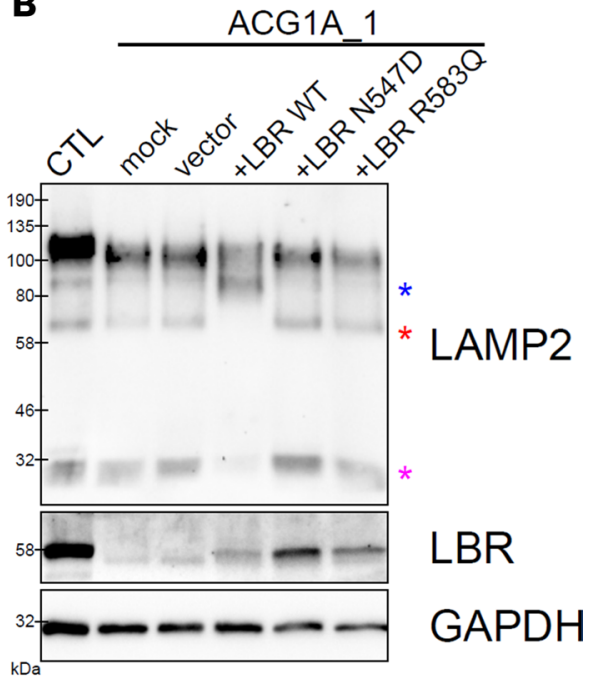

Figure 8. LBR sterol reductase activity restores glycan processing in LBR-deficient cells. (A) Immunofluorescence microscopy of ACG1A_1 fibroblasts stained with LBR-specific antibodies; ACC1A_1 carries the homozygous LBR-null mutation. Cells were either not transfected (parental) or transiently transfected with vectors overexpressing the indicated LBR variants; the Greenberg dysplasia-associated p.N547D and p.R583Q missense mutations specifically abrogate C14 sterol reductase activity but do not alter the chromatin/lamin-binding properties of the respective LBR protein. WT, wild type. Scale bar: $10 \mu \mathrm{m}$. (B) ACG1A_1 cells were either electroporated without plasmid (mock), with empty vector (vector), or with expression constructs encoding the indicated LBR proteins.

Whole-cell protein lysates of nontransfected wild-type controls (CTL) and ACG1A_1 transfectants were obtained 96 hours after electroporation and analyzed by Western blotting with LBR- and LAMP2-specific antibodies. GAPDH immunostaining of the stripped membrane indicates total protein loading. Fast-migrating nonglycosylated LAMP2 is highlighted by a pink asterisk at approximately $30 \mathrm{kDa}$; intermediate glycosylation products are marked by a red asterisk at approximately $65 \mathrm{kDa}$. In ACG1A_1 cells overexpressing wild-type LBR, nonglycosylated LAMP2 and intermediate glycosylation products are not detectable and mature LAMP2 has a similar molecular weight to that in nontransfected wild-type fibroblasts with an additional intermediate band at approximately 85 $\mathrm{kDa}$ (blue asterisk), likely corresponding to a more highly modified species than that seen upon LBR deficiency, although not fully mature. Note that a higher protein load of nontransfected wild-type fibroblasts was used to visualize the normal electrophoretic mobility pattern of LAMP2 species.

\section{Discussion}

Ever since its initial discovery as a lamin-binding nuclear receptor almost 30 years ago (34), LBR has remained an enigmatic protein. This is primarily due to the highly distinct and seemingly incompatible properties of LBR as a structural component of the inner nuclear membrane and an enzyme with specialized tasks in the biosynthesis of sterols $(3,49)$. Another reason for the unresolved physiological role of LBR is the lack of a genuine in vivo model that allows deduction of normal function from the knockout phenotype. To date, no animal model faithfully recapitulates human developmental anomalies associated with LBR inactivation (14-16). Recessive loss-of-function mutations of LBR are known to cause GRBGD, a prenatally lethal chondrodysplasia with recognizable radiographic features $(1,50)$. The most discriminative individual findings in GRBGD include the "moth-eaten" appearance of scapular and pelvic bones and ectopic calcifications of cartilage and connective tissues that, in spite of some phenotypic variability, were present in all 4 confirmed cases to date (50). GRBGD is considered to be the null phenotype of $L B R$ mutations, whereas variants that are predicted to retain residual functions cause milder skeletal phenotypes (Supplemental Table 1) $(6,7)$. However, only few studies have characterized the effect of disease-associated mutation on $L B R$ in detail (8, $12,13)$, as GRBGD is an extremely rare disease and patient-derived materials are scarce. GRBGD-associated $L B R$ mutations may cause alterations of neutrophil granulocyte nuclear morphology in healthy heterozygous carriers (PHA) and a similar phenotype was also seen in $L b r$-mutant mice $(15,16)$. Yet, PHA is not seen in all GRBGD carriers and it remains an open question whether the $L B R$-associated nuclear lobulation defects of hematopoietic cells are due to haploinsufficiency, or caused by a dominant-negative effect of some LBR variants $(5,51)$. As the C14 sterol reductase activity of LBR is redundant in Lbr-knockout mice (14), both dominant and recessive LBR-associated diseases, as well as harmless PHA, were proposed to result from structural defects of the nuclear envelope and were classified as envelopathies (4). However, this notion remains controversial and has recently been challenged by a comprehensive study of GRBGD-causing mutations that demonstrated a nonredundant metabolic function of LBR for cholesterol biogenesis in human cells (12, 13, 49). Significantly, all studied disease-associated variants had no, or severely reduced, C14 reductase activity 


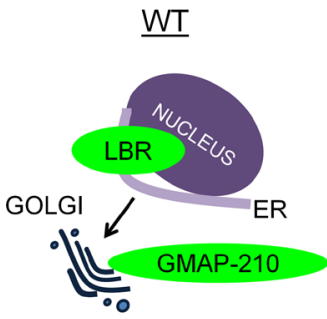

normal cargo secretion

+ glycosylation

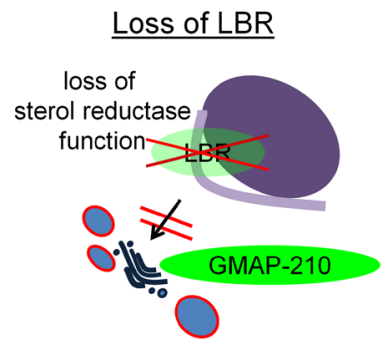

reduced cholesterol
Loss of GMAP-210

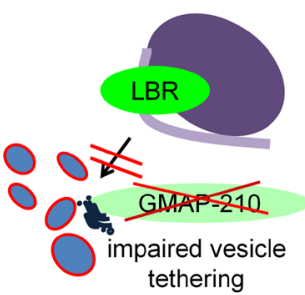

reduced tethering

impaired vesicular trafficking

+ Golgi fragmentation

$\downarrow$

reduced cargo secretion + altered glycosylation

$\downarrow$

ACG1A

Figure 9. LBR and GMAP-210 deficiency impair vesicular transport in the early secretory pathway and cause defective glycoprocessing by the Golgi apparatus. Left: Wild-type cells. Middle: LBR localizes to the nuclear envelope and to the endoplasmic reticulum (ER), functioning in lamin/chromatin-binding and as a sterol reductase. Loss of LBR impairs de novo synthesis of cholesterol and causes disruption of cholesterol-sensitive ER/Golgi trafficking, resulting in defective posttranslational glycan processing and Golgi vesiculation. Right: GMAP-210 is a tether for transport vesicles at the cis-Golgi and is required to maintain Golgi structure. Loss of GMAP-210 also leads to disruption of ER/Golgi trafficking, impaired glycan processing, and Golgi vesiculation. Thus, both lesions converge on Golgi organization and function in achondrogenesis 1A (ACC1A).

and were unable to rescue the cells' dependency on exogenous cholesterol supply. The authors of this study further uncovered that truncated LBR protein variants lacking the carboxy-terminal enzymatic domain are actively eliminated at the nuclear envelope by a novel protein degradation pathway (12). Although these findings are highly significant and imply that GRBGD is in fact a metabolic disorder of cholesterol biosynthesis, the pathogenesis of developmental anomalies in LBR-associated diseases remains unknown.

Using WES, we unexpectedly identified a homozygous c. $[366+1 G>T] L B R$ splice site mutation in a fetus with the unambiguous clinical-radiographic diagnosis of ACG1A; all distinctive signs of GRBGD were missing in our case $(1,50)$. Prior to unbiased disease gene identification by WES trio analysis, the only known molecular cause of ACG1A and possible differential diagnoses had been excluded by Sanger sequencing. Molecular characterization of $L B R$ in patient-derived primary cells proved a fully penetrant missplice of exon 3 , resulting in a frameshift and early termination codon in the mutant ORF (LBR p.E112S $\left.f s^{*} 39\right)$. LBR mRNA was barely detectable by real-time quantitative RT-PCR, compatible with NMD (27). Accordingly, both Western blotting and immunofluorescence studies demonstrated that fetal cells were completely devoid of any LBR protein; hence, the ACG1A case described here represents to our knowledge the first experimentally proven LBR-null phenotype.

Why does a complete loss of LBR result in a disease other than GRBGD? Comparing the known mutational spectrum of GRBGD (Human Gene Mutation Database) (52) with our case offers a straightforward explanation; homozygous GRBGD-associated truncations or missense mutations are all located downstream of exon 11, which preserves the N-terminal nucleoplasmic domain of LBR (encoded by exons 2 to 5 of $L B R$, Supplemental Figure 7 and Supplemental Table 1). In the single compound heterozygous GRBGD case, an early stop variant was only found in combination with a mutation affecting the $3^{\prime}$ end of $L B R$ (Supplemental Figure 7 and Supplemental Table 1). Thus, shortened dysfunctional, or misfolded missense full-length LBR peptides comprising the lamin-interacting domain are predicted in every known confirmed GRBGD case. Recent evidence suggests that carboxy-terminal truncations render some mutant LBR proteins metabolically unstable and lead to rapid proteasomal degradation at the inner nuclear membrane (12). In contrast, the ACG1A-associated $L B R$ c. $[366+1 \mathrm{G}>\mathrm{T}]$ mutation disrupts all annotated protein-coding transcript variants, with the exception of a very short putative variant of 51 amino acids (Supplemental Figure 7). We therefore hypothesize that, in addition to a loss of $\mathrm{C} 14$ reductase activity, the evolution of the distinct GRBGD disease phenotype may depend on remnant LBR protein species. Aberrant LBR proteins are translocated and actively degraded in the nucleus, thereby causing a yet to be characterized cellular (stress) response (12). 
However, the effect of splice mutations in general is context dependent and may not be fully penetrant (53). Although we did not detect any correctly spliced mRNA in ACG1A fibroblasts, the homozygous $L B R$ c. $[366+1 G>T]$ mutation may leave trace amounts of functional LBR in other cell types of the fetus (e.g., in chondrocytes), which may modify the GRBGD phenotype.

LBR and GMAP-210 are proteins with distinct intracellular localizations, different functions, and no known functional or physical interaction. How can mutations in unrelated genes then result in the same phenotype? We hypothesized that the similarity of developmental defects indicated a common downstream pathology and screened patient-derived primary cells for shared morphological abnormalities by deep phenotyping. In our approach, we first focused on GMAP-210-deficient cells, as there are known subcellular abnormalities of the ER and the Golgi apparatus in GMAP-deficient mice and cells (17, 23, 28, 42). However, although there is general agreement about a Golgi localization of GMAP-210 in mammals, several functions have been proposed for the protein $(21,54,55)$. In particular, there is conflicting evidence regarding GMAP-210 involvement in protein trafficking and Golgi organization $(21-23,28)$. Importantly, whereas the ER morphology of ACG1A-derived primary cells had a variable and so far inconclusive phenotype, depending on the underlying genetic defect, our observations confirm that GMAP-210 is indeed absolutely required for Golgi organization in humans. Golgi ultrastructure was severely disrupted in TRIP11-mutant cells and compartment-specific markers demonstrated that loss of GMAP-210 not only affected the cis-Golgi, but caused compaction of the whole organelle. Our results are in line with 2 recent studies investigating the consequences of GMAP-210 depletion in generic cell models $(23,28)$. Efficient knockdown of GMAP-210 caused an identical Golgi compaction phenotype in HeLa cells, with ribbon fragmentation, and a drastic reduction of cisternal number and length (28), indicating that GMAP-210 is also a nonredundant structural component of the Golgi in human cells derived from other tissues. However, the most significant discovery of our study was that all ACG1A-derived primary cells exhibited a common Golgi vesiculation phenotype that was not only previously seen in GMAP-210-depleted HeLa cells (28), but in Gmap-210-deficient mouse chondrocytes as well (17, 42). In GMAP-210-depleted human cells, Golgi vesiculation is accompanied by trafficking defects in the early secretory pathway (23). We observed equivalent secretory trafficking defects in patient-derived fibroblasts, consistent with GMAP-210 acting as a rate-limiting tether for transport vesicles at the cis-Golgi apparatus. Indeed, vesicle formation and turnover is sensitive to any kind of change in GMAP-210 abundance, as not only the depletion, but also the overexpression of GMAP-210 in human and insect cells causes Golgi vesiculation and a block in secretory trafficking $(24,56)$. Our findings support a nonredundant golgin tether function of GMAP-210 for vesicular protein trafficking in human cells that likely is of particular relevance for cell types with high secretion rates, and/or complex cargoes, like ECM proteoglycans produced by chondrocytes.

While the accumulation of transport vesicles seems a plausible consequence of losing an essential Golgi tether protein, it is not obvious why a similar phenotype should ensue from impaired LBR function. However, de novo synthesis of cholesterol is compromised in LBR-mutant cells $(10,12,13)$, and it has been demonstrated that alterations in cholesterol levels can induce Golgi vesiculation $(44,57)$. Along these lines, several publications demonstrated a reduction of ER-to-Golgi protein transport upon intracellular cholesterol depletion by conditioned media or pharmacological inhibition of de novo biosynthesis (58, 59). Structurally, cholesterol depletion leads to dispersal of Golgi cisternae (60) and although it is less well understood how, altered cholesterol levels in intracellular compartments may have an impact on soluble $N$-ethylmaleimide-sensitive-factor attachment receptor (SNARE) localization and function, which in turn could influence secretory trafficking kinetics (61). Thus, while the exact mechanism by which cholesterol impacts upon secretory trafficking has still to be unraveled, we conclude that both LBR and GMAP-210 deficiency converge on impaired vesicular transport in the early secretory pathway, most likely due to altered vesicle-membrane interactions at the ER-Golgi interface. For LBR-associated diseases, we believe our findings are novel and infer a fundamentally different pathogenesis than currently assumed (Figure 9).

How does a secretory trafficking block of the Golgi apparatus impact on skeletal development and lead to a specific achondrogenesis phenotype? Initial results from Trip11-knockout mice suggested that a reduced cargo flux predominantly affects cell types and tissues of the developing skeleton that have high secretion rates for ECM proteins (17). Yet, constitutive ablation of Trip11 results in perinatal lethality, so more subtle or later disease manifestations in other tissues may have been missed. Based on recent results from conditional inactivation in mice, a limited role of Gmap-210 for cargoes exclusively secreted by chondrocytes was proposed (42). In keeping with previous results obtained in generic cell models, our findings support a broader role of GMAP-210 for secretory trafficking in humans $(23,28)$. Consistent with a generally impaired 
transit of secretory proteins through the Golgi, our findings confirm a severe glycosylation defect of Golgi-processed cargo in both TRIP11- and LBR-mutant primary human fibroblasts. Thus, incorrectly modified proteins will at least partially contribute to abnormal differentiation and ossification of skeletal tissues seen in ACG1A. This view is supported by the fact that a mere deficiency in proteoglycan modification causes a phenotypically overlapping lethal chondrodysplasia, ACG1B (62). In keeping with the ACG1A mouse model $(17,42)$, we hypothesize that the striking similarity of LBR- and GMAP-210-associated ACG1A may be rather based on aberrant posttranslational processing of a limited number of secreted developmental regulators with key importance for early chondrogenesis, but this clearly awaits further elucidation. We further propose that a cholesterol-dependent secretory protein trafficking defect is an underlying pathogenic mechanism that causes developmental anomalies in LBR-associated disorders. This also carries implications for other disorders of the CDP spectrum that are caused by enzyme deficiencies in the sterol biosynthesis pathway (11). Of note, skeletal defects in X-linked chondrodysplasia punctata 2 (CDPX2, MIM 3022960), due to loss-of-function mutations in the gene encoding delta(8)-delta(7)sterol isomerase emopamil-binding protein $(E B P)$, may be highly similar to $\operatorname{GRBGD}(29,63)$, which again might point to a common Golgi-related pathogenesis in a genetically heterogeneous disease group. Another important inference of our observations is that impaired vesicular trafficking in the secretory pathway and defective glycoprocessing may be amenable to intervention by cholesterol loading, or other pharmacological strategies to influence intracellular lipid composition. Targeting intracellular cholesterol transport mechanisms has already proved to be a successful therapeutic strategy to modify clinical manifestations in other disease contexts $(58,64)$.

In conclusion, we demonstrate here that mutations in LBR or TRIP11 cause a common clinical and cellular phenotype that is based on impaired secretory trafficking and aberrant glycan processing by the Golgi apparatus. A glycosylation deficit is potentially central to ACG1A skeletal pathophysiology, giving reason to pursue therapeutic strategies in the future to restore normal glycosylation. Our results emphasize the importance of the Golgi complex for the developing skeleton and imply a Golgi-based disease mechanism for GRBGD and a number of related chondrodysplasias with until now unresolved pathophysiology. Further elucidation of the molecular circuits of sterol metabolism, vesicular transport, and protein modification in ACG1A and GRBGD promises exclusive insight into basic cellular mechanisms.

\section{Methods}

Mutation analysis. DNA was obtained from fibroblasts by standard extraction procedures (Qiagen). For the genomic analysis and mutation detection, exons including intron-exon boundaries of TRIP11 and LBR were amplified by PCR and analyzed on an ABI3130xl capillary sequencer (Life Technologies) as described previously $(8,17)$. Bioinformatics allele frequency analysis was based on an in-house exome database, the NHLBI Exome Sequencing Project (http://evs.gs.washington.edu/EVS/), the 1000 Genomes Project (http://www. internationalgenome.org/), and the dbSNP database (https://www.ncbi.nlm.nih.gov/projects/SNP/).

WES. Exome sequences were enriched from DNA using the SureSelect Exome Enrichment kit V4 (Agilent Technologies). Sequencing was performed on a 5500xl SOLiD System (Life Technologies), producing 75-bp single-end reads. For subsequent bioinformatics analysis, an in-house pipeline based on GATK best practice was used (65). Alignments were generated by the proprietary Lifescope software (66). Variant files were annotated using the variant effect predictor software (67) and processed using GEMINI (68), with filter parameters set for allele frequencies of $\leq 1 \%$ in the 1000 Genomes Project, an impact severity classification of medium or high, and a recessive inheritance pattern.

Cell culture. Human fibroblasts were derived from skin biopsies and grown at $37^{\circ} \mathrm{C}$ and $5 \% \mathrm{CO}_{2}$ in Dulbecco's modified Eagle Medium (DMEM) supplemented with 10\% (vol/vol) fetal bovine serum (FBS), 1 mM L-glutamine, 1\% nonessential amino acids (NEAAs, Thermo Fisher Scientific; alanine, asparagine, aspartic acid, glycine, glutamic acid, proline, and serine at $0.1 \mathrm{mM}$ final concentration), $100 \mathrm{IU} / \mathrm{ml}$ penicillin and $100 \mu \mathrm{g} / \mathrm{ml}$ streptomycin (1\% [vol/vol] PenStrep, Thermo Fisher Scientific).

Plasmids and transfection. ACG1A_1 fibroblasts were seeded on $10-\mathrm{cm}$ dishes at $80 \%$ confluence the day before transfection in complete medium lacking antibiotics. After 24 hours, $1 \times 10^{6}$ cells were cotransfected in $200 \mu$ electroporation buffer (Bio-Rad, Gene Pulser buffer, 165-2676) with $2 \mu \mathrm{g}$ pEGFP-N1 (Clontech) and $20 \mu \mathrm{g}$ of pCDNA3.1/V5-His TOPO vector (Invitrogen) or of the respective expression plasmids LBR WT, LBR N547D, and LBR R583Q (12) (a gift from Pei-Ling Tsai, Yale University, New Haven, Connecticut, USA) using a Gene Pulser Xcell electroporation system (Bio-Rad) with a preset protocol for primary fibroblasts according to the manufacturer's recommendations. Green-fluorescent cells 
were sorted on a fluorescence-activated cell sorter (FACS Aria Fusion, Becton Dickinson) 24 hours after transfection; 200,000 EGFP-positive ACG1A_1 cells were collected for each transfectant, plated in 6-well plates, and cultured for an additional 72 hours in complete growth medium before subsequent analysis.

Quantification of cell viability and proliferation. Triplicates of controls and patient-derived fibroblasts were seeded either on 96 -well plates (5,000 cells/well), or 12-well plates (30,000 cells/well) and grown in DMEM supplemented with 10\% (vol/vol) lipoprotein-depleted FBS (S5394, Sigma-Aldrich), $1 \mathrm{mM}$ L-glutamine, 1\% NEAAs, and 1\% penicillin-streptomycin mix, or lipid-restrictive medium supplemented with SyntheChol NS0 Supplement (S5442, Sigma-Aldrich; 1:1,000 final dilution, corresponding to $6.5 \mu \mathrm{M}$ cholesterol in aqueous solution). Day 0 values were determined 4 hours after plating. Supernatants were then removed and adherent cells quantified by crystal violet on a Tecan plate reader at $590 \mathrm{~nm}$ as described previously (69). Numbers of viable cells for a crystal violet dye reference curve and for those grown in 12 -well plates were automatically counted after trypsinization by a Muse cell analyzer (Merck Millipore) as described by the manufacturer.

Cholesterol depletion of fibroblasts. Cells were seeded on $3.5-\mathrm{cm}$ dishes and grown in complete medium until they reached $80 \%$ confluence and then incubated for 48 hours in serum-free DMEM supplemented with $2.5 \mathrm{mM} \mathrm{M} \beta \mathrm{CD}$ or $2.5 \mathrm{mM} \mathrm{M} \beta \mathrm{CD}$ :cholesterol-saturated complexes. $\mathrm{M} \beta \mathrm{CD}$ :cholesterol complexes were prepared as described previously (30). Briefly, cholesterol was dissolved in a mixture of chloroform and methanol $(2: 1, \mathrm{vol} / \mathrm{vol})$ to make a $50 \mathrm{mg} / \mathrm{ml}$ stock solution. Sixteen microliters of the solution was put into a glass vial and nitrogen gas was used to evaporate the solvent, creating a thin film of cholesterol, which was dissolved in $2.5 \mathrm{mM}$ M $\beta C D$ prepared in serum-free DMEM. The solution was further sonicated for 30 minutes in an ultrasonic bath, incubated overnight at $37^{\circ} \mathrm{C}$ with agitation, and filtered through a $0.22-\mu \mathrm{m}$ syringe filter prior to use. Images were collected on an Olympus IX83 inverted microscope using a 20×/0.45 LUC PlanFL N objective with phase-contrast and captured using an Orca ER camera (Hamamatsu) through CellSens software (Olympus). Images were then processed and analyzed using ImageJ (NIH).

Sterol analysis of cultured cells. Human fibroblasts were grown at $37^{\circ} \mathrm{C}$ and $5 \% \mathrm{CO}_{2}$ for 5 days in $10 \%$ (vol/vol) lipoprotein-deficient serum (S5394, Sigma-Aldrich) supplemented with 1\% NEAAs and 1\% penicillin-streptomycin mix. Cells were harvested by centrifugation, washed 3 times with PBS, sonicated, lysed in TNE buffer (50 mM Tris- $\mathrm{HCl}$ [pH 7.5], $20 \mathrm{mM} \mathrm{NaCl}, 0.1 \mathrm{mM}$ EDTA) and extracted with methanol/chloroform. After silylation, alkaline hydrolysis and derivatization with $N, O$-bis(trimethylsilyl)trifluoroacetamide (BSTFA), samples were analyzed by GC/MS using a triple quadrupole mass spectrometer (Agilent 6460 QQQ); $5 \beta$-cholestan-3 $\alpha$-ol was used as internal standard.

Pulse-chase analysis of protein secretion. Analysis of protein secretion was performed using a pulse-chase approach as described previously (45). Cells were seeded onto $3.5-\mathrm{cm}$ dishes and grown until they reached $95 \%$ confluence. Cells were starved for 1 hour in methionine- and cysteine-free DMEM (Invitrogen) supplemented with $10 \%$ (vol/vol) FBS and $1 \mathrm{mM} \mathrm{L-glutamine}$ and pulsed for 20 minutes with fresh starvation medium containing $50 \mu \mathrm{Ci} / \mathrm{ml}\left[{ }^{35} \mathrm{~S}\right] \mathrm{Met}$ and $\left[{ }^{35} \mathrm{~S}\right]$ Cys protein-labeling mix (PerkinElmer). After washing with $\mathrm{PBS}$, cells were incubated in chase medium (DMEM, $10 \mathrm{mM}$ HEPES pH 7.4, $1 \mathrm{mM}$ methionine, $1 \mathrm{mM}$ cysteine, $100 \mu \mathrm{g} / \mathrm{ml}$ bovine serum albumin) for 0,30 , or 60 minutes. Proteins secreted to the medium were precipitated using trichloroacetic acid (TCA), while cells were lysed using HMNT lysis buffer. Cells and medium fractions were subjected to SDS-PAGE, gels were fixed for 10 minutes in 20\% (vol/vol) methanol and 10\% (vol/vol) acetic acid, and dried for 2 hours at $65^{\circ} \mathrm{C}$ using a gel dryer (Bio-Rad). Dried gels were exposed to phosphorimaging plates and developed after 10 days using a fluorescent image analyzer (FLA 7000, Fujifilm). Signals from radiolabeled proteins were quantified using AIDA software (Elysia-Raytest).

$R N A$ extraction, reverse transcription $P C R$, and quantitative $P C R$. Total RNA from fibroblasts and cell lines was extracted with TRIzol (Invitrogen), followed by DNase I treatment (Roche) and column purification (Qiagen). RNA was quantified by spectrophotometry (Nanodrop ND-8000, peqlab), and cDNA was synthesized from $1 \mu \mathrm{g}$ total RNA with Superscript III reverse transcriptase (Invitrogen) and oligo dT16 primers. Quantitative PCR was performed on a CFX384Touch Real-Time PCR System (Bio-Rad). Delta cycle threshold (Ct) relative quantification, PCR efficiency correction, and multiple reference gene normalization (UBC, TBP, HMBS, and $P D H A$ ) were calculated with qBase (Bio-Rad). Oligonucleotides for TRIP11 and $L B R$ were designed using the NCBI Primer Blast software (https://www.ncbi.nlm.nih.gov/tools/primer-blast/). Primer specificity was verified by agarose gel electrophoresis, Sanger sequencing of PCR products, and melting curve analysis.

Antibodies. The following antibodies were used: rabbit anti-LBR (ProteinTech, 12398-1-AP), goat antilamin B (C-20; Santa Cruz Biotechnology), mouse anti-ERGIC-53 (G1/93; Enzo Life Sciences), rabbit 
anti-GMAP-210 (HPA002570, Sigma-Aldrich), mouse anti-p230 (clone 15; BD Biosciences), rabbit antiPDI (SPA-890; Stressgen Biotechnologies), rabbit anti-Sec13 (Rainer Pepperkok, European Molecular Biology Laboratory, Heidelberg, Germany), sheep anti-ZFPL1 (70), rabbit anti-giantin (Manfred Renz, Institute of Immunology and Molecular Genetics, Karlsruhe, Germany), mouse anti-GAPDH (G-9; Santa Cruz Biotechnology), rabbit anti-LAMP-1 (D2D11, Cell Signaling Technology), and anti-LAMP2 (H4B4; Developmental Studies Hybridoma Bank, University of Iowa, Iowa City, Iowa, USA). Alexa 488-, 546-, 594-, and 647-conjugated secondary antibodies were from Molecular Probes (Thermo Fisher Scientific). Horseradish peroxidase-conjugated secondary antibodies were from Sigma-Aldrich.

Immunoblotting. Fibroblasts were lysed using either radioimmunoprecipitation assay buffer (RIPA; 20 $\mathrm{mM}$ Tris- $\mathrm{HCl}$ [pH 7.5], $150 \mathrm{mM} \mathrm{NaCl}, 1 \mathrm{mM} \mathrm{Na} \mathrm{FDTA}_{2} 1 \mathrm{mM}$ EGTA, 1\% NP-40, 1\% sodium deoxycholate, $2.5 \mathrm{mM}$ sodium pyrophosphate, and $1 \mathrm{mM} \beta$-glycerophosphate) supplemented with dithiothreitol (DTT, Bio-Rad), benzonase (Merck Millipore), protease inhibitor cocktail (Calbiochem), PhosStop (Roche), or HMNT lysis buffer supplemented with protease inhibitor cocktail; $30 \mu \mathrm{g}$ of cell lysates was separated by SDS-PAGE. Proteins were then transferred onto nitrocellulose membranes (Amersham Hybond 0.45 NC, GE Healthcare) using a wet transfer system. The efficiency of transfer was assessed by Ponceau S stain (Sigma-Aldrich). Membranes were blocked by incubation in PBST buffer (PBS with $0.1 \%$ Tween 20) supplemented with 5\% skim milk for 1 hour at room temperature (RT) and incubated with primary antibody solution (5\% skim milk in PBST) overnight at $4^{\circ} \mathrm{C}$. Membranes were washed 3 times in PBST buffer, for 5 minutes each at RT (all subsequent steps at RT), and incubated with secondary antibody solution ( $5 \%$ skim milk in PBST) for 1 hour. Membranes were washed 3 times in PBST buffer for 5 minutes and once in PBS for 5 minutes. The immunoreaction was detected with SuperSignal West Pico ECL chemiluminescent substrate (Thermo Fisher Scientific) using a Bio-Rad Chemidoc MP Imager. Data were analyzed using Image Lab software (Bio-Rad).

Glycan processing analysis of fibroblasts. For the analysis of cholesterol-dependent protein glycosylation, cells were seeded on $3.5-\mathrm{cm}$ dishes and grown in complete medium until they reached $80 \%$ confluence and then incubated for 24 or 48 hours in serum-free DMEM supplemented with $1.25 \mathrm{mM} \mathrm{M \beta CD}$. Cells were lysed using HMNT lysis buffer (20 mM HEPES-KOH pH 7.4, $5 \mathrm{mM} \mathrm{MgCl}, 0.1 \mathrm{M} \mathrm{NaCl}, 0.5 \%$ [wt/vol] Triton X-100) supplemented with protease inhibitor cocktail (Calbiochem) and $30 \mu \mathrm{g}$ of cell lyses was subjected to SDS-PAGE, as described above, and blotted with anti-LAMP2 antibody.

Fluorescence microscopy. Fibroblasts were seeded on glass coverslips and grown to $90 \%$ confluence. Cells were washed twice with PBS, fixed with 3\% (wt/vol) paraformaldehyde (PFA; Sigma-Aldrich) in PBS for 25 minutes at RT. Cells were then washed with PBS and excess PFA was quenched with glycine. Cells were permeabilized by 4-minute incubation in $0.1 \%$ (wt/vol) Triton X-100 in PBS. For ER morphology analysis, cells were fixed for 20 minutes at RT in a fixative solution composed of 3\% (wt/vol) PFA and 0.025\% (wt/vol) glutaraldehyde (GA; electron microscopy-grade, Sigma-Aldrich). Cells were then washed 3 times with PBS. PFA-GA was quenched by washing 3 times in freshly prepared $1 \mathrm{mg} / \mathrm{ml} \mathrm{NaBH}_{4}$ solution in $\mathrm{PBS}$ for 5 minutes at RT followed by 3 washes with PBS. Cells were then permeabilized by 4 -minute incubation in $0.1 \%$ (wt/vol) Triton X-100 and $0.05 \%$ (wt/vol) SDS in PBS. Cells were incubated with primary antibody diluted in PBS for 1 hour at RT and incubated 3 times with PBS for 5 minutes. Then coverslips were incubated for 1 hour with secondary antibody diluted in PBS, sometimes together with $100 \mathrm{ng} / \mathrm{ml}$ Hoechst 33342 dye (Thermo Fisher Scientific) to stain DNA, and incubated 3 times with PBS for 5 minutes and twice in $\mathrm{ddH}_{2} 0$ for 5 minutes. Coverslips were dried before mounting in Mowiol 4-88. Images were acquired using an Olympus BX60 upright microscope equipped with a MicroMax cooled, slow-scan CCD camera (Princeton Instruments) driven by Metaview software (University Imaging Corporation). Images were processed using ImageJ software.

Electron microscopy. Cells were grown on $10-\mathrm{cm}$ dishes until they reached $80 \%$ confluence. The samples were fixed with $4 \%$ PFA plus $2.5 \%$ GA in 0.1 M HEPES buffer ( $\mathrm{pH} 7.2$ ). Fixed cells were scraped and centrifuged for 10 minutes at $5,200 \mathrm{~g}$. They were then postfixed with $1 \%$ osmium tetroxide plus $1.5 \%$ potassium ferrocyanide in $0.1 \mathrm{M}$ cacodylate buffer $(\mathrm{pH}$ 7.2) for 1 hour and in $1 \%$ uranyl acetate in water for 1 hour. The postfixed samples were dehydrated in ethanol series infiltrated with Low Viscosity resin (TAAB) and polymerized for 24 hours at $60^{\circ} \mathrm{C}$. Sections $(70 \mathrm{~nm}$ ) were cut with an Ultracut ultramicrotome (Reichert) and observed with an FEI Tecnai 12 Biotwin microscope at $100 \mathrm{kV}$ accelerating voltage. Images were obtained with a Gatan Orius SC1000 CCD camera. Quantification of cells with large vesicular profiles, defined as peri-Golgi circular profiles with a diameter of $>100 \mathrm{~nm}$, were conducted manually in a blind manner.

Statistics. Statistical analyses were conducted using GraphPad Prism. D'Agostino-Pearson and Shapiro-Wilk tests were used for comparison of the distribution of data with a Gaussian distribution. Depending on 
the result, a Mann-Whitney test, 1-way or 2-way ANOVA with Bonferroni's correction for multiple comparisons was performed. The number of cells with large vesicular profiles was compared using a $\chi^{2}$ test. Amounts of secreted proteins were compared using 1-way ANOVA followed by Dunnett's multiple comparisons test. Statistical significance cutoffs were set as follows: ${ }^{*} P \leq 0.05$, ${ }^{* *} P<0.01,{ }^{* * *} P<0.001$, and ${ }^{* * * *} P<0.0001$.

Study approval. All individuals in our study were recruited by physician-initiated referral. The study was conducted in accordance with the Declaration of Helsinki protocols and approved by the institutional ethics review board of Freiburg University Hospital, Germany (Ethik-Kommission der Albert-Ludwigs-Universität Freiburg Nr. 349/13). Written informed consent for skin biopsies and molecular studies was obtained from the parents, or legal guardians of included cases in accordance with current German law (GenDG). Control samples and primary cells were collected from matched healthy individuals under the same criteria and regulations.

\section{Author contributions}

EL and ML designed the research studies. AW, TMW, and JCS conducted experiments and acquired data. AW, TMW, JCS, SB, JS, AH, AK, ME, EL, and ML contributed and analyzed data. AW and EL wrote the manuscript. AW, TMW, EL, and ML edited the manuscript. All other co-authors read, commented on, and approved the manuscript.

\section{Acknowledgments}

This work was supported by grants from the German Research Foundation (CRC1140, project B6 to EL, project A5 to AK), and by the European Commission (SYBIL consortium grant agreement no. 602300, Rarenet [a project of INTERREG V]) to EL, and by an MRC research grant (MR/N000366/1) awarded to ML. The authors wish to thank Aleksandr Mironov for the assistance in electron microscopy and the Wellcome Trust for equipment grant support to the EM Facility at the University of Manchester. The Bioimaging Facility microscopes used in this study were purchased with grants from BBSRC, Wellcome, and the University of Manchester Strategic Fund. We gratefully acknowledge Pei-Ling Tsai and Christian Schlieker (Yale University, New Haven, Connecticut, USA), who provided us with expression constructs for LBR.

Address correspondence to: Martin Lowe, Faculty of Biology, Medicine and Health, University of Manchester, The Michael Smith Building, Oxford Road, Manchester M13 9PT, United Kingdom. Phone: 44.161.275.5387; Email: martin.lowe@manchester.ac.uk. Or to: Ekkehart Lausch, Department of Pediatrics, Medical Centre-University of Freiburg, Mathildenstrasse 1, D-79106 Freiburg, Germany. Phone: 49.761.270.43630; Email: ekkehart.lausch@uniklinik-freiburg.de.

1. Spranger J, Maroteaux P. The lethal osteochondrodysplasias. Adv Hum Genet. 1990;19:1-103.

2. Bonafe L, et al. Nosology and classification of genetic skeletal disorders: 2015 revision. Am J Med Genet A. 2015;167A(12):2869-2892.

3. Nikolakaki E, Mylonis I, Giannakouros T. Lamin B receptor: Interplay between structure, function and localization. Cells. 2017;6(3):E28.

4. Janin A, Bauer D, Ratti F, Millat G, Méjat A. Nuclear envelopathies: a complex LINC between nuclear envelope and pathology. Orphanet J Rare Dis. 2017;12(1):147.

5. Colella R, Hollensead SC. Understanding and recognizing the Pelger-Huët anomaly. Am J Clin Pathol. 2012;137(3):358-366.

6. Sobreira N, et al. An anadysplasia-like, spontaneously remitting spondylometaphyseal dysplasia secondary to lamin B receptor (LBR) gene mutations: further definition of the phenotypic heterogeneity of LBR-bone dysplasias. Am J Med Genet A. 2015;167A(1):159-163.

7. Borovik L, Modaff P, Waterham HR, Krentz AD, Pauli RM. Pelger-huet anomaly and a mild skeletal phenotype secondary to mutations in LBR. Am J Med Genet A. 2013;161A(8):2066-2073.

8. Hoffmann K, et al. Mutations in the gene encoding the lamin B receptor produce an altered nuclear morphology in granulocytes (Pelger-Huët anomaly). Nat Genet. 2002;31(4):410-414.

9. Greenberg CR, Rimoin DL, Gruber HE, DeSa DJ, Reed M, Lachman RS. A new autosomal recessive lethal chondrodystrophy with congenital hydrops. Am J Med Genet. 1988;29(3):623-632.

10. Waterham HR, et al. Autosomal recessive HEM/Greenberg skeletal dysplasia is caused by 3 beta-hydroxysterol delta 14 -reductase deficiency due to mutations in the lamin B receptor gene. Am J Hum Genet. 2003;72(4):1013-1017.

11. Waterham HR, Ebberink MS. Genetics and molecular basis of human peroxisome biogenesis disorders. Biochim Biophys Acta. 2012;1822(9):1430-1441.

12. Tsai PL, Zhao C, Turner E, Schlieker C. The Lamin B receptor is essential for cholesterol synthesis and perturbed by disease-causing mutations. Elife. 2016;5:e16011.

13. Clayton $\mathrm{P}$, et al. Mutations causing Greenberg dysplasia but not Pelger anomaly uncouple enzymatic from structural functions of a nuclear membrane protein. Nucleus. 2010;1(4):354-366.

14. Wassif CA, et al. HEM dysplasia and ichthyosis are likely laminopathies and not due to 3beta-hydroxysterol Delta14-reductase 
deficiency. Hum Mol Genet. 2007;16(10):1176-1187.

15. Shultz LD, et al. Mutations at the mouse ichthyosis locus are within the lamin B receptor gene: a single gene model for human Pelger-Huët anomaly. Hum Mol Genet. 2003;12(1):61-69.

16. Verhagen AM, et al. Reduced lymphocyte longevity and homeostatic proliferation in lamin B receptor-deficient mice results in profound and progressive lymphopenia. J Immunol. 2012;188(1):122-134.

17. Smits P, et al. Lethal skeletal dysplasia in mice and humans lacking the golgin GMAP-210. N Engl J Med. 2010;362(3):206-216.

18. Grigelioniene $\mathrm{G}$, et al. The phenotype range of achondrogenesis 1A. Am J Med Genet A. 2013;161A(10):2554-2558.

19. Witkos TM, Lowe M. The golgin family of coiled-coil tethering proteins. Front Cell Dev Biol. 2015;3:86.

20. Gillingham AK, Munro S. Finding the Golgi: Golgin coiled-coil proteins show the way. Trends Cell Biol. 2016;26(6):399-408

21. Ríos RM, Sanchís A, Tassin AM, Fedriani C, Bornens M. GMAP-210 recruits gamma-tubulin complexes to cis-Golgi membranes and is required for Golgi ribbon formation. Cell. 2004;118(3):323-335.

22. Yadav S, Puri S, Linstedt AD. A primary role for Golgi positioning in directed secretion, cell polarity, and wound healing. Mol Biol Cell. 2009;20(6):1728-1736.

23. Roboti P, Sato K, Lowe M. The golgin GMAP-210 is required for efficient membrane trafficking in the early secretory pathway. J Cell Sci. 2015;128(8):1595-1606.

24. Pernet-Gallay K, Antony C, Johannes L, Bornens M, Goud B, Rios RM. The overexpression of GMAP-210 blocks anterograde and retrograde transport between the ER and the Golgi apparatus. Traffic. 2002;3(11):822-832.

25. Zankl A, et al. Preselection of cases through expert clinical and radiological review significantly increases mutation detection rate in multiple epiphyseal dysplasia. Eur J Hum Genet. 2007;15(2):150-154.

26. Desmet FO, Hamroun D, Lalande M, Collod-Béroud G, Claustres M, Béroud C. Human Splicing Finder: an online bioinformatics tool to predict splicing signals. Nucleic Acids Res. 2009;37(9):e67.

27. Kurosaki T, Maquat LE. Nonsense-mediated mRNA decay in humans at a glance. J Cell Sci. 2016;129(3):461-467.

28. Sato K, Roboti P, Mironov AA, Lowe M. Coupling of vesicle tethering and Rab binding is required for in vivo functionality of the golgin GMAP-210. Mol Biol Cell. 2015;26(3):537-553.

29. Offiah AC, et al. Greenberg dysplasia (HEM) and lethal X linked dominant Conradi-Hünermann chondrodysplasia punctata (CDPX2): presentation of two cases with overlapping phenotype. J Med Genet. 2003;40(12):e129.

30. Christian AE, Haynes MP, Phillips MC, Rothblat GH. Use of cyclodextrins for manipulating cellular cholesterol content. $J$ Lipid Res. 1997;38(11):2264-2272.

31. Luchetti G, et al. Cholesterol activates the G-protein coupled receptor Smoothened to promote Hedgehog signaling. Elife. 2016;5:e20304.

32. Appelbaum J, Blobel G, Georgatos SD. In vivo phosphorylation of the lamin B receptor. Binding of lamin B to its nuclear membrane receptor is affected by phosphorylation. J Biol Chem. 1990;265(8):4181-4184

33. Worman HJ, Evans CD, Blobel G. The lamin B receptor of the nuclear envelope inner membrane: a polytopic protein with eight potential transmembrane domains. J Cell Biol. 1990;111(4):1535-1542.

34. Worman HJ, Yuan J, Blobel G, Georgatos SD. A lamin B receptor in the nuclear envelope. Proc Natl Acad Sci USA. 1988;85(22):8531-8534.

35. Ye Q, Worman HJ. Primary structure analysis and lamin B and DNA binding of human LBR, an integral protein of the nuclear envelope inner membrane. J Biol Chem. 1994;269(15):11306-11311.

36. Herrmann H, Zwerger M. The danger of "multi-tasking”: LBR out of control. Nucleus. 2010;1(4):319-324.

37. Zwerger M, Kolb T, Richter K, Karakesisoglou I, Herrmann H. Induction of a massive endoplasmic reticulum and perinuclear space expansion by expression of lamin B receptor mutants and the related sterol reductases TM7SF2 and DHCR7. Mol Biol Cell. 2010;21(2):354-368.

38. Rios RM, et al. A peripheral protein associated with the cis-Golgi network redistributes in the intermediate compartment upon brefeldin A treatment. J Cell Biol. 1994;125(5):997-1013.

39. Perri ER, Thomas CJ, Parakh S, Spencer DM, Atkin JD. The unfolded protein response and the role of protein disulfide isomerase in neurodegeneration. Front Cell Dev Biol. 2015;3:80.

40. Hauri HP, Kappeler F, Andersson H, Appenzeller C. ERGIC-53 and traffic in the secretory pathway. J Cell Sci. 2000;113(Pt 4):587-596.

41. Schweizer A, Fransen JA, Bächi T, Ginsel L, Hauri HP. Identification, by a monoclonal antibody, of a 53-kD protein associated with a tubulo-vesicular compartment at the cis-side of the Golgi apparatus. J Cell Biol. 1988;107(5):1643-1653.

42. Bird IM, et al. The skeletal phenotype of achondrogenesis type 1A is caused exclusively by cartilage defects. Development. 2018;145(1):dev156588.

43. Stüven E, et al. Intra-Golgi protein transport depends on a cholesterol balance in the lipid membrane. J Biol Chem 2003;278(52):53112-53122.

44. Grimmer S, Iversen TG, van Deurs B, Sandvig K. Endosome to Golgi transport of ricin is regulated by cholesterol. Mol Biol Cell. 2000;11(12):4205-4216.

45. Roboti P, Witkos TM, Lowe M. Biochemical analysis of secretory trafficking in mammalian cells. Methods Cell Biol. 2013;118:85-103.

46. Schwake M, Schröder B, Saftig P. Lysosomal membrane proteins and their central role in physiology. Traffic. 2013;14(7):739-748.

47. Carlsson SR, Roth J, Piller F, Fukuda M. Isolation and characterization of human lysosomal membrane glycoproteins, h-lamp-1 and h-lamp-2. Major sialoglycoproteins carrying polylactosaminoglycan. J Biol Chem. 1988;263(35):18911-18919.

48. Li J, Pfeffer SR. Lysosomal membrane glycoproteins bind cholesterol and contribute to lysosomal cholesterol export. Elife. 2016;5:e21635.

49. Turner EM, Schlieker C. Pelger-Huët anomaly and Greenberg skeletal dysplasia: LBR-associated diseases of cholesterol metabolism. Rare Dis. 2016;4(1):e1241363.

50. Konstantinidou A, et al. Pathologic, radiographic and molecular findings in three fetuses diagnosed with HEM/Greenberg skeletal dysplasia. Prenat Diagn. 2008;28(4):309-312.

51. Hoffmann K, Sperling K, Olins AL, Olins DE. The granulocyte nucleus and lamin B receptor: avoiding the ovoid. Chromosoma. 
2007;116(3):227-235.

52. Stenson PD, et al. The Human Gene Mutation Database: towards a comprehensive repository of inherited mutation data for medical research, genetic diagnosis and next-generation sequencing studies. Hum Genet. 2017;136(6):665-677.

53. Grünert SC, et al. Medium-chain acyl-CoA dehydrogenase deficiency associated with a novel splice mutation in the ACADM gene missed by newborn screening. BMC Med Genet. 2015;16:56.

54. Broekhuis JR, Rademakers S, Burghoorn J, Jansen G. SQL-1, homologue of the Golgi protein GMAP210, modulates intraflagellar transport in C. elegans. J Cell Sci. 2013;126(Pt 8):1785-1795.

55. Follit JA, et al. The Golgin GMAP210/TRIP11 anchors IFT20 to the Golgi complex. PLoS Genet. 2008;4(12):e1000315.

56. Friggi-Grelin F, Rabouille C, Therond P. The cis-Golgi Drosophila GMAP has a role in anterograde transport and Golgi organization in vivo, similar to its mammalian ortholog in tissue culture cells. Eur J Cell Biol. 2006;85(11):1155-1166.

57. Hansen GH, Niels-Christiansen LL, Thorsen E, Immerdal L, Danielsen EM. Cholesterol depletion of enterocytes. Effect on the Golgi complex and apical membrane trafficking. J Biol Chem. 2000;275(7):5136-5142.

58. Runz H, et al. Inhibition of intracellular cholesterol transport alters presenilin localization and amyloid precursor protein processing in neuronal cells. J Neurosci. 2002;22(5):1679-1689.

59. Ridsdale A, Denis M, Gougeon PY, Ngsee JK, Presley JF, Zha X. Cholesterol is required for efficient endoplasmic reticulum-to-Golgi transport of secretory membrane proteins. Mol Biol Cell. 2006;17(4):1593-1605.

60. Xu Y, Takeda S, Nakata T, Noda Y, Tanaka Y, Hirokawa N. Role of KIFC3 motor protein in Golgi positioning and integration. J Cell Biol. 2002;158(2):293-303.

61. Enrich C, Rentero C, Hierro A, Grewal T. Role of cholesterol in SNARE-mediated trafficking on intracellular membranes. J Cell Sci. 2015;128(6):1071-1081.

62. Superti-Furga A, et al. Achondrogenesis type IB is caused by mutations in the diastrophic dysplasia sulphate transporter gene Nat Genet. 1996;12(1):100-102.

63. Irving MD, Chitty LS, Mansour S, Hall CM. Chondrodysplasia punctata: a clinical diagnostic and radiological review. Clin Dysmorphol. 2008;17(4):229-241.

64. Trushina E, Canaria CA, Lee DY, McMurray CT. Loss of caveolin-1 expression in knock-in mouse model of Huntington's disease suppresses pathophysiology in vivo. Hum Mol Genet. 2014;23(1):129-144.

65. DePristo MA, et al. A framework for variation discovery and genotyping using next-generation DNA sequencing data. Nat Genet. 2011;43(5):491-498.

66. Pranckevičiene E, Rančelis T, Pranculis A, Kučinskas V. Challenges in exome analysis by LifeScope and its alternative computational pipelines. BMC Res Notes. 2015;8:421.

67. McLaren W, et al. The Ensembl variant effect predictor. Genome Biol. 2016;17(1):122.

68. Paila U, Chapman BA, Kirchner R, Quinlan AR. GEMINI: integrative exploration of genetic variation and genome annotations. PLoS Comput Biol. 2013;9(7):e1003153.

69. Feoktistova M, Geserick P, Leverkus M. Crystal violet assay for determining viability of cultured cells. Cold Spring Harb Protoc. 2016;2016(4):pdb.prot087379.

70. Chiu CF, et al. ZFPL1, a novel ring finger protein required for cis-Golgi integrity and efficient ER-to-Golgi transport. EMBO J. 2008;27(7):934-947 